\title{
Boundary operators in effective string theory
}

\author{
Simeon Hellerman ${ }^{a}$ and Ian Swanson \\ ${ }^{a}$ Kavli Institute for the Physics and Mathematics of the Universe, The University of Tokyo, \\ Kashiwa, Chiba 277-8582, Japan \\ E-mail: simeon.hellerman.1@gmail.com, ianswanson.physics@gmail.com
}

ABSTRACT: Various universal features of relativistic rotating strings depend on the organization of allowed local operators on the worldsheet. In this paper, we study the set of Neumann boundary operators in effective string theory, which are relevant for the controlled study of open relativistic strings with freely moving endpoints. Relativistic open strings are thought to encode the dynamics of confined quark-antiquark pairs in gauge theories in the planar approximation. Neumann boundary operators can be organized by their behavior under scaling of the target space coordinates $X^{\mu}$, and the set of allowed $X$-scaling exponents is bounded above by $+1 / 2$ and unbounded below. Negative contributions to $X$-scalings come from powers of a single invariant, or "dressing" operator, which is bilinear in the embedding coordinates. In particular, we show that all Neumann boundary operators are dressed by quarter-integer powers of this invariant, and we demonstrate how this rule arises from various ways of regulating the short-distance singularities of the effective theory.

KEYwords: 1/N Expansion, Bosonic Strings, Effective field theories

ArXIV EPRINT: 1609.01736 


\section{Contents}

1 Introduction 1

2 Heuristic motivation $\quad 4$

2.1 Dressing rules for bulk and Dirichlet boundary operators 4

2.2 The Neumann dressing rule 5

$\begin{array}{lll}3 & \text { Effective strings from perturbed Liouville theory } & 7\end{array}$

$\begin{array}{lll}3.1 & \text { Origin of the dressing rule for bulk operators } & 7\end{array}$

$\begin{array}{lll}3.2 & \text { Demonstration of the boundary dressing rule } & 10\end{array}$

$\begin{array}{lll}3.3 & \text { From UV operators to effective boundary operators } & 13\end{array}$

$\begin{array}{ll}3.4 & \text { Quantum corrections leave the boundary dressing rule unmodified }\end{array}$

$\begin{array}{lll}4 & \text { A displaced-boundary regulator } & \mathbf{1 5}\end{array}$

$\begin{array}{lll}\text { 4.1 Definition of the displaced-boundary regulator scheme } & 17\end{array}$

$\begin{array}{lll}4.2 & \text { Boundary operators } & 19\end{array}$

$\begin{array}{lll}4.3 & \text { Bulk operators } & 21\end{array}$

4.4 Anomaly term 22

5 Conclusions and physical consequences $\quad 23$

A Calculation of the asymptotic Regge intercept 25

$\begin{array}{ll}\text { B Properties of geodesics near the boundary } & 30\end{array}$

\section{Introduction}

For many purposes, it is useful to study the dynamics of relativistic strings in the framework of a Wilsonian effective field theory on the string worldsheet. This enterprise is known as effective string theory. A manifestly Poincaré-invariant version of this theory was invented in [1]. This theory was more recently elucidated by embedding it into the Polyakov formalism $[2,3]$, which simplifies the construction of vertex operators and the renormalization of short-distance singularities of the worldsheet path integral. This simplified covariant formalism has been applied to calculate subleading terms in the perturbative expansion of the mass-squared of rotating string states at large angular momentum $J$ [4].

Open relativistic strings with freely moving endpoints are of particular interest, as these objects are believed to describe the dynamics of confined quark-antiquark pairs in gauge theories in the planar approximation. In the covariant formalism, the condition of freely moving endpoints corresponds to a Neumann boundary condition on the embedding coordinates $X^{\mu}$, at leading order in the expansion around large angular momentum $J$. To 
analyze higher-order corrections and renormalize such theories correctly, it is important to characterize the spectrum of boundary operators in the open worldsheet theory with Neumann boundary conditions, as has been done [5-7] for operators in the interior of the worldsheet and for boundary operators with Dirichlet boundary conditions. ${ }^{1}$

In all cases, local operators are organized hierarchically under an assignment of $X$ scaling dimension, which encodes the scaling dimension of the term in the standard Wilsonian sense. (We use the term " $X$-scaling dimension" to distinguish the $X$-scaling from the scaling dimensions of operators under the residual Virasoro symmetry of the Polyakov formalism after gauge-fixing the metric. The Virasoro algebra is a residual gauge symmetry of the Polyakov action, and the weights of all physical states and operators under it are determined by gauge symmetry.) The spectrum of $X$-scaling dimensions of operators depends on the details of the particular effective string theory under consideration, but in all cases is bounded above, and continues discretely downwards towards $-\infty$. Negative $X$-scalings come from negative powers of the "dressing" operator, a distinguished bilinear invariant of $X$, which compensates the conformal scaling dimension of a nonsingular numerator. The numerators are polynomials in derivatives of $X$.

For bulk operators and Dirichlet boundary operators, the operator spectrum is organized according to a rule for dressing operators for each case, specifying the unique operator that can occur to negative or fractional powers, and which fractional exponents can occur. In the case of bulk operators in conformal gauge, the dressing rule amounts to the condition that operators are dressed with negative integer powers of the dressing operator $\mathcal{I}_{11}$, where ${ }^{2}$

$$
\mathcal{I}_{p q} \equiv \partial_{+}^{p} X \cdot \partial_{-}^{q} X .
$$

For boundaries where some coordinates $X$ have Neumann boundary conditions and some have Dirichlet, the dressing rule for boundary operators is that the operator dressing consists only of half-integer powers of $\mathcal{B}_{(11)} \equiv \dot{X}_{\text {NEUMANN }}^{2}$, where we introduce the notation

$$
\mathcal{B}_{(p q)} \equiv \partial_{\sigma^{0}}^{p} X \cdot \partial_{\sigma^{0}}^{q} X
$$

for boundary operators. In each case there exist a number of simple motivations for the dressing rule, which we shall discuss briefly in section 2. For instance, the correct result for the dressing rule can be stated in terms of a pure power principle: the dressing comes in negative powers of the most relevant bilinear invariant operator. In the example above, the dressing consists of powers of the operator $\mathcal{O}=\mathcal{B}_{(11)}^{1 / 2}$.

The dressing rule in the case of Neumann boundary conditions is equally simple but less familiar. We will show below that at Neumann boundaries, operators are dressed with negative quarter-integer powers of the operator $\mathcal{B}_{(22)}$, or, equivalently, negative integer powers of the operator $\mathcal{O}_{\text {(quark })} \equiv \mathcal{B}_{(22)}^{1 / 4}$, which encodes the leading physical effect of an infinitesimal change in the mass of the quark. This is another instance of the pure power principle: the operator $\mathcal{O}_{\text {(quark) }}$ is the invariant with the largest $X$-scaling in the theory with Neumann boundaries.

\footnotetext{
${ }^{1}$ For strings with all spacelike directions satisfying Dirichlet boundary conditions, the organization of operators has been supported by numerical evidence from lattice simulations [8-11].

${ }^{2}$ Our worldsheet coordinate conventions are $\sigma^{ \pm} \equiv \sigma^{0} \pm \sigma^{1}=\tau \pm \sigma, \partial_{ \pm}=\frac{1}{2}\left(\partial_{0} \pm \partial_{1}\right)$.
} 
The dressing rule for Neumann boundary operators is particularly physically significant, because there is an anomaly term in the Lagrangian density with a universal coefficient $^{3}$ that has a non-integrable divergence near a Neumann boundary. This singularity does not signify a breakdown of the effective theory. Rather, the singularity is removed by a familiar procedure of short-distance regularization, and the divergence in the quantum effective action when the regulator is removed must be cancelled by a boundary counterterm. In this case the counterterm is the quark mass operator $\mathcal{O}_{\text {(quark) }}$ itself [4]. Thus, demonstrating the renormalizability of the effective string theory with Neumann boundary conditions depends on the use of the correct dressing rule.

Moreover, the universality of the large- $J$ spectrum (and other observables) at relative order $J^{-1}$ also depends on the use of the correct dressing rule: there are neither bulk nor boundary terms in the action with adjustable coefficients at order $|X|^{0}$ (which is order $|X|^{-2}$ relative to the Nambu-Goto action). Amplitudes are therefore universal at relative order $J^{-1}$, since the length of a string scales with its angular momentum as (length) $\sim J^{\frac{1}{2}}$. If there were such an operator at order $|X|^{0}$, amplitudes would not be universal at relative order $J^{-1}$. Demonstrating the absence of order- $|X|^{0}$ operators at Neumann boundaries, and therefore the universality of the asymptotic intercept of the Regge trajectory, thus depends on the correct derivation and application of the dressing rule.

The goal of the paper is to explain the origin of the dressing rule for boundary operators on strings with freely moving endpoints. In a covariant gauge, in which all $D$ embedding coordinates appear on equal footing as dynamical worldsheet fields, the leading-order boundary condition is that all $D$ coordinates obey Neumann conditions $\partial_{\text {normal }} X^{\mu}=0$ at the boundary. For concreteness, we will work in the simplified covariant formalism introduced in [3], with the $($ diff $) \times\left(\right.$ Weyl) symmetry fixed by taking unit gauge $g_{a b}=\eta_{a b}$. However this is only to simplify the discussion: all other known consistent formalisms and gauges for the effective string, can be obtained by gauge-fixing the simplified covariant formalism.

We will work within the context of the simplified covariant formalism introduced in [3], which includes an independent intrinsic metric $g_{a b}$ transforming under a Weyl symmetry $g_{a b} \rightarrow \exp \{2 \rho\} g_{a b}$. The anomaly in the gauge symmetry is compensated by an explicit anomaly term. The Nambu-Goto string is recovered from the (partial) gauge fixing condition that sets $g_{a b}$ equal to the induced metric $g_{a b} \rightarrow \partial_{a} X \cdot \partial_{b} X$, which fixes the Weyl symmetry while leaving the diffeomorphism symmetry unfixed. In this (partial) gaugefixing, the anomaly term does not need to be added and its physical effect appears in the quantum effective action. In the standard unit gauge $g_{a b} \rightarrow \eta_{a b}$, its effect appears through an explicit term in the bare action. Unit gauge simplifies calculations considerably in practice, and we shall always structure our derivations with unit gauge in mind. The dressing rule in other gauges can be obtained straightforwardly by gauge-fixing the simplified-covariant expressions.

\footnotetext{
${ }^{3}$ We are using the term "universal" according to its usual meaning in the context of effective field theory, i.e., that the value of the asymptotic intercept does not depend on the values of adjustable parameters in the effective worldsheet action. If one considers a universality class with different low-energy degrees of freedom or symmetries, the value of the asymptotic intercept may of course differ.
} 
To provide some context, and to guide the subsequent analysis, we will begin in the following section by motivating the dressing rule on heuristic grounds. In section 3 we will provide a more concrete derivation of the dressing rule starting from an ultraviolet-complete worldsheet theory. As a demonstration that the conclusions are in fact independent of the details of any particular UV completion of the effective string theory, we take yet another approach in section 4. There, we adopt a displaced-boundary UV regulator scheme and show how the dressing rule emerges from this regulator for a few specific operators of interest. We conclude in section 5 with a broader discussion of some of the physical consequences of the dressing rule. As supplementary material to both the study of boundary operators in effective string theory, and to the larger goal of understanding certain aspects of the strong-coupling dynamics of QCD via string theory, we provide in the appendices a more detailed and fully gauge-invariant calculation of the asymptotic Regge intercept (a result originally presented in [4]), as well as other calculational details that support the conclusions drawn in the main body of the paper.

\section{Heuristic motivation}

We have proposed a boundary operator dressing rule in effective string theory, which states that at Neumann boundaries, operators are dressed with negative quarter-integer powers of the operator $\mathcal{B}_{(22)}$. In this section we will motivate this claim on heuristic grounds, based on minimality, naturalness and on the pure power principle discussed above. Following this, we will turn to a more detailed derivation of the Neumann-boundary dressing rule.

\subsection{Dressing rules for bulk and Dirichlet boundary operators}

Minimality. We begin with an overview of bulk worldsheet operators and Dirichlet boundary operators. In ref. [1], the dressing rule for bulk operators was assumed rather than derived. There, Polchinski and Strominger (PS) introduced a singular interaction term in the Lagrangian of order $|X|^{0}$ for spacetime dimension $D \neq 26$ :

$$
\mathcal{L}_{\mathrm{PS}}=\frac{\beta}{2 \pi} \frac{\mathcal{I}_{12} \mathcal{I}_{21}}{\mathcal{I}_{11}^{2}},
$$

which compensates for the conformal anomaly by contributing $\Delta c=26-D$ to the central charge of the conformal dynamics of the embedding coordinates $X^{\mu}$. Rather than basing the form of this term explicitly on general restrictions descending from the symmetries and constraints of the theory, the authors arrived at eq. (2.1) based on the specific requirement that the conformal anomaly cancel for small perturbations around the static string solution. The denominator of the singular term is $\mathcal{I}_{11}^{2}$, from which it follows that integer powers of $\mathcal{I}_{11}$ must be allowed in the bulk operator dressing rule. Singularities in operator products between powers of the anomaly term above have denominators that are integer powers of $\mathcal{I}_{11}$ only. To all orders in large- $|X|$ perturbation theory, then, the rule that bulk operators appear dressed strictly with integer powers of $\mathcal{I}_{11}$ is stable against quantum corrections. An $\mathcal{I}_{11}$ dressing rule can thus be taken as the minimal rule that is consistent with anomaly cancellation and stable against quantum corrections. It is this notion of minimality that we will propose be applied to the case of Neumann boundaries. 
Naturalness and genericity: the pure power principle. For bulk operators and Dirichlet boundary operators, the dressing operator is the unique monomial with the lowest $X$-scaling obeying the required symmetries, and it is automatic that such an operator is a conformal tensor. In other words, the dressing rule for bulk and Dirichlet operators takes the form of negative integer powers of the most relevant operator that can be expressed as a power of a bilinear invariant. Below we will promote this structure to an ansatz for dressing operators in general, and apply it to the case of Neumann boundaries.

As discussed briefly in [3], the bulk dressing rule follows in some sense from naturalness. If an operator of nonmarginal conformal weight is dressed to conformality with some other operator, and we assume the latter is generic (in the sense of being a linear combination of all possible operators of the appropriate conformal weight), then at large $J$ the dressing operator will be dominated by powers of a single operator, $\mathcal{I}_{11}$ (that is, the operator that contains the most powers of $X$ per conformal dimension). The same conclusion holds for mixed Dirichlet-Neumann boundaries, such as those describing strings with fixed, static endpoints: for mixed Dirichlet-Neumann boundary conditions, ${ }^{4}$ naturalness dictates that the dressing operator be dominated by powers of $\mathcal{B}_{(11)}$. For comparison, with strictly Dirichlet boundary conditions we expect the dressing rule should be formulated purely in powers of $\left(\dot{X}^{\prime}\right)^{2}$.

We will generalize this notion of naturalness to the case of Neumann boundaries as well. However, as a final comment, it is worth noting that it is a logical possibility that there may exist other types of boundary conditions or singularities where there is not a unique leading large- $X$ invariant operator, and thus no unique candidate for a dressing operator. In these cases it would clearly be more difficult, for instance, to arrive at universality statements regarding terms arising in the perturbative analysis of the physical spectrum.

\subsection{The Neumann dressing rule}

Let us now proceed with a proposal for an operator dressing rule at Neumann boundaries that satisfies the same properties as the bulk and Dirichlet dressing rules summarized above. To motivate the claim on heuristic grounds, we begin with the ansatz that our dressing operator be a monomial in bilinears in derivatives of the embedding coordinates $X$. To satisfy the principle of naturalness, we require the monomial to have the highest possible $X$-scaling for its worldsheet conformal dimension (or, equivalently, the lowest possible worldsheet conformal dimension for its $X$-scaling). Both $X$-scaling and (in the semiclassical regime) conformal dimension are additive under multiplication, so the ratio of $X$-scaling to worldsheet conformal dimension is maximized by powers of a single bilinear. Therefore,

\footnotetext{
${ }^{4}$ The distinction between "mixed Dirichlet-Neumann" and "purely Dirichlet" is in some sense gaugedependent. In static gauge, where the time direction is not an independent variable, an infinitely heavy static quark is represented by a "purely Dirichlet" boundary condition on the unconstrained degrees of freedom, i.e., the transverse Goldstone bosons. In conformal gauge, the same boundary condition is mixed, because the timelike embedding coordinate $X^{0}$ is treated as an independent degree of freedom before restricting to the physical Hilbert space, and so takes Neumann boundary conditions. The case of purely Dirichlet boundary conditions in covariant gauge is potentially relevant to gauge instantons in planar QCD, and can't even in principle be described in static gauge.
} 
we will identify the nonvanishing bilinear with lowest possible worldsheet conformal scaling dimension and see that it is unique up to operator equivalences.

First, one can use the equations of motion to reduce all derivatives of $X$ to the form $\partial_{0}^{p} X$ or $\partial_{0}^{p} \partial_{1} X$, where Neumann boundary conditions can be used to eliminate the latter. ${ }^{5}$ The only invariant candidate operators remaining are then of the form

$$
\mathcal{B}_{(p q)}=\partial_{0}^{p} X \cdot \partial_{0}^{q} X
$$

introduced in (1.2). It is important to note here that when we eliminate other possible operators from this search, we are only doing so modulo operators of lower $X$-scaling. For the purpose of estimating leading-order scalings and proceeding order-by-order in $J$, this is always sufficient, and whenever we refer to eliminating operators by appealing to constraints or to the equations of motion, we will always mean it in this sense.

The operator $\mathcal{B}_{(11)}$ is proportional to the leading-order stress tensor, and thus vanishes as an operator, modulo operators of lower $X$-scaling. The operator $\mathcal{B}_{(12)}$ is a tangential derivative of $\mathcal{B}_{(11)}$, and also vanishes, modulo operators of lower $X$-scaling. Next we come to the operators $\mathcal{B}_{(22)}$ and $\mathcal{B}_{(13)}$. The sum of these is proportional to a second tangential derivative of $\mathcal{B}_{(11)}$, and we can eliminate $\mathcal{B}_{(13)}$ as an operator in favor of $\mathcal{B}_{(22)}$, modulo operators of lower $X$-scaling.

The operator $\mathcal{B}_{(22)}$ itself is an independent, gauge-invariant operator. For instance, it takes a nonzero expectation value, proportional to $J$, in the lowest state of angular momentum $J$ that satisfies the Virasoro constraints. It is natural to propose, then, that $\mathcal{B}_{(22)}$ should be the dressing operator for effective string theories with Neumann boundaries. More precisely, and following the pure-power principle, the proposed dressing rule states that the basis for symmetry-preserving boundary operators in conformal gauge, with Neumann boundary conditions, is

$$
\mathcal{O} \in \operatorname{Span}\left\{\mathcal{B}_{(22)}^{-\frac{1}{4}(\Delta-1)} \cdot \prod_{i} \mathcal{B}_{\left(p_{i} q_{i}\right)} ; \quad \Delta \equiv \sum_{i} p_{i}+q_{i}\right\} .
$$

Before moving on to a more principled derivation of this rule, we pause to make some final comments about the proposed $\mathcal{B}_{(22)}$ dressing rule for Neumann boundaries:

- Ordinarily, we expect that perturbations of the Lagrangian in conformal gauge must be Virasoro primaries of weight one. The operator $\mathcal{B}_{(22)}^{a}$ is not Virasoro primary; the lowering operator $L_{1}$ does not annihilate it identically, but gives an operator proportional to $\mathcal{B}_{(12)} \mathcal{B}_{(22)}^{a-1}$. The operator $\mathcal{B}_{(12)}$ vanishes on the constraint space, so the quark-mass term $\mathcal{I}_{(22)}^{1 / 4}$ is gauge-invariant in matrix elements between states satisfying the constraint. In order to see the gauge-invariance of the term off the constraint space, one could define the boundary perturbation in a fully conformally invariant way by starting with the Weyl-invariant $\widehat{\mathcal{I}}_{22} \equiv \mathcal{I}_{22}-\left(\mathcal{I}_{12} \mathcal{I}_{21}\right) / \mathcal{I}_{11}$, which is a conformal tensor, and taking it to the boundary. The fully $($ diff $) \times($ Weyl)-invariant version of $\widehat{\mathcal{I}}_{(22)}$ in the simplified covariant formalism is given in reference [3]. For our purposes this is not necessary.

\footnotetext{
${ }^{5}$ Where we now replace explicit worldsheet directions $\sigma^{0,1}$ with $(0,1)$ indices.
} 
- The rule has direct phenomenological consequences: the leading gauge-invariant boundary operator $\mathcal{B}_{(22)}$ has been studied in other gauges $[12,13]$, and the insertion of its fourth root into the boundary action corresponds to the leading effect of an infinitesimal variation of the quark mass at large $J$.

- Finally, in actual UV-complete worldsheet theories, where the non-Goldstone degrees of freedom can be integrated out, the dressing rule for Neumann boundaries in the effective theory is indeed the one we have described. In what follows, we will demonstrate this in full detail in one such UV completion of the effective theory, taking the form of a perturbed Liouville theory [3]. We will also comment in more detail on how this rule satisfies the minimality principle outlined above.

\section{Effective strings from perturbed Liouville theory}

We have now motivated the dressing rule for Neumann boundaries on heuristic grounds. Namely, Neumann boundary operators are dressed with negative quarter-integer powers of the operator $\mathcal{B}_{(22)}$. In this section we derive this statement rigorously from the starting point of a particular UV complete worldsheet theory.

In [1], Polchinski and Strominger present a microscopic model from which an effective string description might emerge, in terms of a $(D+1)$-dimensional string theory with a Liouville direction. At large $D$, the PS-Liouville Lagrangian looks like

$$
\mathcal{L}=\frac{|D|}{24 \pi}(\partial \phi)^{2}+\mu^{2} e^{-2 \phi}+\mu^{\prime-2} e^{2 \phi} \mathcal{I}_{11}^{2}+(\phi-\text { independent }) .
$$

Here, $\mu$ and $\mu^{\prime}$ are arbitrary mass parameters. One can introduce open strings into this model by introducing a space-filling brane into the Liouville theory.

\subsection{Origin of the dressing rule for bulk operators}

We begin our analysis of this theory by extracting the form of the dressing rule for bulk operators noted above. The intent is that this will provide context for the subsequent analysis in the boundary theory. In particular, we derive the $\mathcal{I}_{11}$ dressing rule for the closed string theory, motivated on general grounds in section 2.1 above. We will derive this dressing rule in a class of UV-complete models that generalizes the construction of ref. [1] to a considerable extent, showing that the dressing rule emerges for completely generic gauge-invariant and Poincaré-invariant operator perturbations of the theory. The ideas in this subsection are to some extent implicit in [1]; we make them explicit to show that they indeed generalize to the case when open strings are included.

First, we redefine the Liouville field in such a way that the second and third terms in the equations of motion scale equally, and the kinetic term for $\phi$ can be neglected in the large- $X$ regime:

$$
\begin{aligned}
e^{\phi} & =\sqrt{\mu \mu^{\prime}} \mathcal{I}_{11}^{-\frac{1}{2}} e^{\hat{\phi}}, \\
\phi & =\hat{\phi}-\frac{1}{2} \log \left(\frac{\mathcal{I}_{11}}{\mu \mu^{\prime}}\right) .
\end{aligned}
$$


Note that this field transformation becomes singular whenever $\mu$ or $\mu^{\prime}$ vanish. This redefinition is specifically adapted to a situation where $\phi$ gets a minimum of its effective potential for a long string, which happens only when both $\mu$ and $\mu^{\prime}$ are nonzero.

Under this field redefinition, the Lagrangian above becomes

$$
\mathcal{L}=\mathcal{L}_{|X|^{2}}+\mathcal{L}_{|X|^{0}}+O\left(|X|^{-2}\right)
$$

with

$$
\begin{aligned}
\mathcal{L}_{|X|^{2}} & \equiv \frac{\mu}{\mu^{\prime}}(\exp (2 \hat{\phi})+\exp (-2 \hat{\phi})) \mathcal{I}_{11}+(\text { terms subleading in } \mathrm{D}) \\
& =\frac{2 \mu}{\mu^{\prime}} \mathcal{I}_{11}+(\text { terms involving } \hat{\phi} \text { fluctuations })+(\text { terms subleading in } \mathrm{D}), \\
\mathcal{L}_{|X|^{0}} & \equiv \mathcal{L}_{\mathrm{PS}}+(\text { terms involving } \hat{\phi} \text { fluctuations })+(\text { terms subleading in } \mathrm{D}) .
\end{aligned}
$$

In particular, the order $|X|^{0}$ term agrees with the PS anomaly Lagrangian up to terms of order $|D|^{0}$, as pointed out in [1].

We observe that the effective theory in [1] can be derived from a much larger class of microscopic models described by perturbations of the $(D+1)$-dimensional string theory with one Liouville direction. Adding higher-derivative terms dressed to conformality with Liouville exponentials leads to the same scaling for $\hat{\phi}$ and the same coefficient for the anomaly term in the effective Lagrangian at leading order. For instance, consider a more general Lagrangian of the form

$$
\begin{aligned}
\mathcal{L} & =\frac{|D|}{24 \pi}(\partial \phi)^{2}+\mu^{2} e^{-2 \phi}+\sum_{q \geq 0} a_{q} \mu^{-2 q} \exp (2 q \phi) \mathcal{I}_{11}^{q+1} \\
& =\frac{|D|}{24 \pi}(\partial \phi)^{2}+\mathcal{I}_{11} F(y)
\end{aligned}
$$

with

$$
y \equiv \mu^{-2} \mathcal{I}_{11} \exp (2 \phi),
$$

where $a_{0}$ is the microscopic string tension (i.e., the string tension in the full $(D+1)$ dimensional string theory), and $a_{1}, a_{2}, \cdots$ are the values of massive stringy condensates. If $\left(y_{0}, F\left(y_{0}\right)\right)$ is the global minimum of $F(y)$, then we can shift the Liouville field to $\hat{\phi}$, where

$$
\begin{aligned}
e^{\phi} & =\mu \sqrt{y_{0}} \mathcal{I}_{11}^{-\frac{1}{2}} e^{\hat{\phi}}, \\
\phi & =\hat{\phi}-\frac{1}{2} \log \left(\frac{\mathcal{I}_{11}}{\mu^{2} y_{0}}\right),
\end{aligned}
$$

whereby we obtain

$$
\begin{aligned}
\mathcal{L} & =\mathcal{L}_{|X|^{2}}+\mathcal{L}_{|X|^{0}}+O\left(|X|^{-2}\right), \\
\mathcal{L}_{|X|^{2}} & \equiv F\left(y_{0}\right) \mathcal{I}_{11}+(\text { terms involving } \hat{\phi} \text { fluctuations })+(\text { terms subleading in } \mathrm{D}), \\
\mathcal{L}_{|X|^{0}} & \equiv \mathcal{L}_{\mathrm{PS}}+(\text { terms involving } \hat{\phi} \text { fluctuations })+(\text { terms subleading in } \mathrm{D}) \cdot
\end{aligned}
$$


Here, the global minimum $F\left(y_{0}\right)$ assumes the role of the effective string tension in the $D$-dimensional effective string theory. The coefficient of the PS anomaly Lagrangian is, as expected, independent of the form of $F(y)$ and in agreement, at leading order in $|D|$, with the value required [1] to compensate the $O(|D|)$ contribution to the central charge deficit.

The terms linear in $\hat{\phi}$ vanish at orders $|X|^{2}$ and $|X|^{1}$; as with (3.2), we have chosen the shift in $\phi(3.7)$ so that $\hat{\phi}=0$ is a solution to the classical equations of motion for $\phi$ with the $O\left(|X|^{0}\right)$ kinetic terms omitted. The mass-squared of the $\hat{\phi}$ fluctuation is of order $|X|^{2}$, and it can be integrated out. The leading contribution of the path integral over $\hat{\phi}$ is of order $|D|^{0}|X|^{0}$, which acts only to shift the coefficient of the Polchinski-Strominger anomaly term by one unit of central charge.

We can now perturb the microscopic string theory with arbitrary gauge-invariant operators and investigate the structure of resulting perturbations of the effective theory. It is immediately clear that the bulk dressing rule is respected. Perturbations of the microscopic theory are generated by monomials in derivatives of $X$ and $\phi$, dressed with Liouville exponentials. But, as we have seen, the classical elimination of the Liouville degree of freedom $\phi$ is such that all Liouville exponentials become powers of $\mathcal{I}_{11}$ in the effective theory.

Now let us consider several sources of error whose discussion we have omitted in the above derivation of the bulk dressing rule; the corresponding corrections will be seen to respect the same dressing rule as the leading terms, in both the large- $D$ and large- $X$ sense.

Corrections to the classical solution for $\phi$. In the above discussion, we shifted $\phi$ so that $\hat{\phi}=0$ is a minimum to accuracy up to and including order $|X|^{-1}$. We can study the effects of the kinetic term order by order in a large- $|X|$ expansion, however. Expanding the classical solution $\phi_{*}$ as $\phi_{*}^{(0)}+\phi_{*}^{(1)}+\cdots$, where $\phi_{*}^{(n)}$ is of order $|X|^{-2 n}$, one can solve for each order iteratively. For instance, in the case of the original PS-Liouville model [1], we have ${ }^{6}$

$$
\phi_{*}^{(1)}=\frac{|D|}{24 \pi} \frac{\mu^{\prime}}{4 \mu} \frac{\partial \bar{\partial} \phi_{*}^{(0)}}{\mathcal{I}_{11}} .
$$

This correction obviously satisfies the dressing rule. More generally, it is straightforward to show inductively that the $n^{\text {th }}$ correction to the classical solution is always of the form

$$
\phi_{*}^{(n)}=P_{n}+\frac{|D|}{24 \pi} \frac{\mu^{\prime}}{4 \mu} \frac{\partial \bar{\partial} \phi_{*}^{(n-1)}}{\mathcal{I}_{11}},
$$

where $P_{n}$ is a polynomial in $\phi_{*}^{(1)}$ through $\phi_{*}^{(n-1)}$, whose total $X$-scaling is exactly $|X|^{-2 n}$. (The polynomials $P_{n}$ come from the large- $|X|$ expansion of the exponentials $\exp \left\{ \pm 2\left(\phi_{*}^{(1)}+\phi_{*}^{(2)}+\cdots\right)\right\}$.) Thus, the dressing rule holds to all orders in the large$|X|$ expansion of the classical solution at large $D$.

In the above, we have used leading-order, large- $D$ expressions for the classical action. At finite $D$, we replace $|D|$ with $26-D$ and supplement $\mathcal{I}_{11}^{2}$ with subleading $D$-dependent terms, proportional to $D^{-1}\left(\partial_{-} X\right)^{2}\left(\partial_{+} X\right)^{2}$, in the form of the irrelevant perturbation.

\footnotetext{
${ }^{6}$ Here, $\partial$ and $\bar{\partial}$ are the usual derivatives with respect to the holomorphic conformal coordinates on the worldsheet.
} 
Such terms are required to make the perturbation a Virasoro primary of weight one (in particular, see eq. (20) of [1]).

Subleading large- $\boldsymbol{D}$ corrections from quantum effects. The large- $D$ regime suppresses quantum corrections to the classical elimination of the $\hat{\phi}$ fluctuations, in terms of contributions to the effective action for the $X$ fields. We can also consider corrections to the Wilsonian action for $X$ in perturbation theory when we integrate out $\hat{\phi}$ at one or more loops. The resulting effective action is of course complicated, but we emphasize that all Feynman diagrams correcting the classical effective action for $X$ give terms obeying the bulk dressing rule.

To see this, let $M_{\hat{\phi}}^{2}$ be the tree-level mass of the $\hat{\phi}$-fluctuation, while $\left\{C_{\hat{\phi}}^{(3,4,5, \cdots)}\right\}$ denote, collectively, its cubic, quartic, quintic, etc., self-couplings. The general structure of the effective action at a given order of perturbation theory will always have elements of $\left\{C_{\hat{\phi}}^{(p)}\right\}$ in the numerator, and powers of $M_{\hat{\phi}}$ in the denominator.

In models (3.1) or (3.5), the mass of the $\hat{\phi}$ fluctuation is exactly proportional to $\mathcal{I}_{11}$, and so the form of the effective action, order by order in perturbation theory, is given by polynomials in $\mathcal{I}_{p q}$, dressed with negative powers of $\mathcal{I}_{11}$. In $1 / D$ perturbation theory, singular operators in the effective action can come only from $\hat{\phi}$-propagators, since the interaction vertices for $\hat{\phi}$ have only positive powers of $X$ in the UV theory. Beyond perturbation theory, the singular $X$-dependence of operators come entirely from the mass scale at which new degrees of freedom enter, namely $M_{\hat{\phi}}^{2} \propto \mathcal{I}_{11}$, plus terms subleading in $|X|$. We therefore infer that the bulk dressing rule holds for all $1 / D$ quantum corrections to the effective string action as well, away from loci on the worldsheet where $\mathcal{I}_{11}$ vanishes.

\subsection{Demonstration of the boundary dressing rule}

We now turn to the boundary theory to demonstrate explicitly the form of the dressing rule for boundary operators in effective string theory, for the Polchinski-Strominger deformed Liouville theory. We do this in the most direct possible way, expanding around a nonsingular classical solution for the Liouville field $\phi$ and integrating out massive fluctuations. The first step is thus to understand how the classical solution for the Liouville field scales in the near-boundary region.

We start by expanding $\mathcal{I}_{11}$ near the boundary:

$$
\mathcal{I}_{11}=-\frac{1}{2} \mathcal{B}_{(22)} \sigma_{1}^{2}+O\left(\sigma_{1}^{3}\right) .
$$

The expansion contains higher terms of the form $\mathcal{B}_{(p q)} \sigma^{p+q-2}$, with $\mathcal{B}_{(p q)}$ defined in eq. (1.2). Such terms are obtained by Taylor expanding $\mathcal{I}_{11}$ near the boundary and using the free EOM and free Virasoro constraints. There are also terms coming from corrections to the free-field EOM due to the interactions with the Liouville field. In this section we will estimate the $J$-scaling of such corrections and the effective terms they generate after the elimination of the Liouville field.

Order by order in $\sigma$, equation (3.11) is an operator statement in the low energy Hilbert space, rather than just a property of a particular classical solution or matrix element in a given state. We now pause to emphasize this distinction. 
In effective string theory, the degrees of freedom are small fluctuations around a lowestenergy classical solution carrying certain conserved global quantum numbers. The lowestlying classical solution carrying a given set of charges is always automatically Virasoroprimary and preserves a 'helical' symmetry, i.e., an invariance under a combined time translation and a global symmetry transformation, which in this context is a rotation of some of the target-space coordinates. (For more details on the helical solution, see eq. (10) of [4], or appendix A.) In the helical solution, the values of the invariants $\mathcal{I}_{p q}$ and $\mathcal{B}_{(p q)}$ are all time-independent, and equation (3.11) is simply an identity between time-independent expectation values. However, the existence of the operator expansion (3.11) does not depend on the helical property: the expansion in fact holds true even for general timedependent perturbations with energies of $O(1)$ above the large- $J$ ground state. For the sake of brevity we are not explicitly indicating any time dependence, though both sides of equation (3.11) can be assumed to depend arbitrarily on $\sigma^{0}$, as consistent with the equations of motion and the Virasoro constraints.

Starting with (3.1), and motivated by (3.11), we invoke the following change of variables

$$
\hat{\hat{\phi}}=\phi+\frac{1}{4} \log \left(\frac{|D|}{24 \pi} \frac{\mathcal{B}_{(22)}}{\mu^{3} \mu^{\prime}}\right), \quad \phi=\hat{\hat{\phi}}-\frac{1}{4} \log \left(\frac{|D|}{24 \pi} \frac{\mathcal{B}_{(22)}}{\mu^{3} \mu^{\prime}}\right)
$$

along with the coordinate rescaling

$$
\hat{\sigma}^{1}=\mathcal{B}_{(22)}^{\frac{1}{4}}\left(\frac{|D|}{24 \pi}\right)^{-\frac{1}{4}} \mu^{\frac{1}{4}} \mu^{\prime-\frac{1}{4}} \sigma^{1}, \quad \sigma^{1} \equiv \mathcal{B}_{(22)}^{-\frac{1}{4}}\left(\frac{|D|}{24 \pi}\right)^{\frac{1}{4}} \mu^{-\frac{1}{4}} \mu^{\prime \frac{1}{4}} \hat{\sigma}^{1} .
$$

As with (3.2), this field redefinition and coordinate transformation make sense only if both $\mu$ and $\mu^{\prime}$ are nonzero. These transformations have been performed so that the unique time-independent classical solution for the shifted field $\hat{\hat{\phi}}$ approaches a fixed limit in the scaling region of fixed $\hat{\hat{\sigma}}_{1}$, as $|X| \rightarrow \infty$.

Of course, we can extend this to a rescaling of both worldsheet coordinates by taking

$$
\hat{\hat{\sigma}}^{0}=\sigma^{0},
$$

so that

$$
\begin{aligned}
& \partial_{1}=\mathcal{B}_{(22)}^{\frac{1}{4}}\left(\frac{|D|}{24 \pi}\right)^{-\frac{1}{4}} \mu^{\frac{1}{4}} \mu^{\prime-\frac{1}{4}} \partial_{\hat{\sigma}^{1}}, \\
& \partial_{0}=\partial_{\hat{\hat{\sigma}}^{0}}+\frac{1}{2} \frac{\mathcal{B}_{(23)}}{\mathcal{B}_{(22)}} \hat{\hat{\sigma}}^{1} \partial_{\hat{\hat{\sigma}}^{1}} .
\end{aligned}
$$

The redefinition of the $\sigma^{0}$ derivative from a fixed $-\sigma_{1}$ to a fixed- $\hat{\hat{\sigma}}_{1}$ partial derivative does not affect the leading $J$-scalings. For instance, we have

$$
\partial_{0} \phi=\partial_{\hat{\hat{\sigma}}^{0}} \hat{\hat{\phi}}-\frac{1}{2} \frac{\mathcal{B}_{(23)}}{\mathcal{B}_{(22)}}+\frac{1}{2} \frac{\mathcal{B}_{(23)}}{\mathcal{B}_{(22)}} \hat{\hat{\sigma}}^{1} \partial_{\hat{\hat{\sigma}}^{1}} \hat{\hat{\phi}}
$$

The latter two terms are order $J^{0}$ and are subleading relative to the $J$-scaling of the $\sigma^{0}$-derivative, which we will see is generally $O\left(J^{\frac{1}{4}}\right)$ (see eq. (3.21)). 
Classical solution. In the classical ground state (i.e., the helical solution), the solution for $\hat{\hat{\phi}}$ is always time-independent:

$$
\partial_{\hat{\hat{\sigma}}_{1}}^{2} \hat{\hat{\phi}}=\frac{\hat{\hat{\sigma}}_{1}^{4}}{4} \exp (2 \hat{\hat{\phi}})-\exp (-2 \hat{\hat{\phi}}) \quad \text { (ground state) } .
$$

The boundary condition for this equation states that $\hat{\hat{\phi}}$ obeys the Neumann condition at $\hat{\hat{\sigma}}_{1}=0$ and continues smoothly to all values of $\sigma_{1}=O(1)$. Let us now define $\hat{\hat{\Phi}}$ as the classical ground state solution for $\hat{\hat{\phi}}$. For $\hat{\hat{\Phi}}^{\prime}(0)=0$, by adjusting the initial value $\hat{\hat{\Phi}}(0)$, it is easy to see that the solution goes to $+\infty$ at finite $\hat{\hat{\sigma}}_{1}$ for $\hat{\hat{\Phi}}(0)>\hat{\hat{\Phi}}^{(\text {crit.) }}(0)$, and to $-\infty$ at finite $\hat{\hat{\sigma}}_{1}$ for $\hat{\hat{\Phi}}(0)<\hat{\hat{\Phi}}^{(\text {crit. })}(0)$, where $\hat{\hat{\Phi}}(0)^{(\text {crit.) }}$ is some critical initial condition lying between the two singular trajectories. In other words, the only value of $\hat{\hat{\Phi}}$ compatible with the boundary condition and the existence of a smooth solution is $\hat{\hat{\Phi}}(0)=\hat{\hat{\Phi}}^{(\text {crit. })}(0)$. It is straightforward to numerically determine the value of $\hat{\hat{\Phi}}^{(\text {crit.) }}(0)$. For the ground-state classical solution,

$$
\hat{\hat{\Phi}}(0)=0.4067
$$

so we find that the full boundary value of $\Phi$ is

$$
\Phi(0)=0.4067-\frac{1}{4} \log \left(\frac{|D|}{24 \pi} \frac{\mathcal{B}_{(22)}}{\mu^{\prime} \mu^{3}}\right)
$$

at large $J$. Note that the specific value (3.18) depends on the details of the perturbation of the Liouville theory. A different form for the perturbation, e.g., a different set of $a_{q}$ in the ansatz parametrized in (3.5), would give the same coefficient of $\log \left(\mathcal{B}_{(22)}\right)$ in equation (3.19), but a different constant term.

Frequencies of normal modes. Now let us estimate the frequencies of normal modes of $\breve{\phi} \equiv \hat{\hat{\phi}}-\hat{\hat{\Phi}}$, localized near the boundary. Such modes are of the form

$$
\breve{\phi}=\exp \left(i \omega \hat{\hat{\sigma}}^{0}\right) f\left(\hat{\hat{\sigma}}^{1}\right)
$$

where the functional dependence of $f\left(\hat{\hat{\sigma}}^{1}\right)$ is fixed in the $J \rightarrow \infty$ limit. At large $J$, the second and third terms in (3.16) are negligible, so the linearized equation of motion for the $\breve{\phi}$ fluctuation can only be satisfied if $\omega$ scales as $J^{\frac{1}{4}}$ :

$$
\omega=O\left(J^{\frac{1}{4}}\right) \text {. }
$$

Now we retain only the leading (first) term in (3.16), and use (3.11) to get a leadingorder action for the shifted Liouville field $\hat{\hat{\phi}}$ in the scaling region $\sigma_{1} \sim \mathcal{B}_{(22)}^{-1 / 4}$. The action (3.1) becomes

$$
\begin{aligned}
\mathcal{L}= & \left(\frac{|D|}{24 \pi}\right)\left(\partial_{\hat{\hat{\sigma}}^{0}} \hat{\hat{\phi}}\right)^{2} \\
& -\left(\frac{\mathcal{B}_{(22)}|D|}{24 \pi}\right)^{\frac{1}{2}}\left(\frac{\mu}{\mu^{\prime}}\right)^{\frac{1}{2}}\left\{\left(\partial_{\hat{\sigma}^{1}} \hat{\hat{\phi}}\right)^{2}+\exp (-2 \hat{\hat{\phi}})+\frac{\hat{\hat{\sigma}}_{1}^{4}}{4} \exp (2 \hat{\hat{\phi}})\right\}+O\left(|X|^{-1}\right) .
\end{aligned}
$$


So, at large $J$, the term $\partial_{0}$ is approximated by $\partial_{\hat{\sigma}^{0}}$, and the equation of motion becomes

$$
\partial_{\hat{\hat{\sigma}}^{0}}^{2} \hat{\hat{\phi}}=\mathcal{B}_{(22)}^{\frac{1}{2}}\left(\frac{|D|}{24 \pi}\right)^{-\frac{1}{2}}\left(\frac{\mu}{\mu^{\prime}}\right)^{\frac{1}{2}}\left\{\partial_{\hat{\hat{\sigma}}^{1}}^{2} \hat{\hat{\phi}}+\exp (-2 \hat{\hat{\phi}})-\frac{\hat{\hat{\sigma}}_{1}^{4}}{4} \exp (2 \hat{\hat{\phi}})\right\} .
$$

If we rewrite quantities in terms of $\hat{\hat{\Phi}}=\hat{\hat{\phi}}-\breve{\phi}$, then the normal mode equation for the mode $f$ in (3.20) takes the form

$$
\omega^{2} f\left(\hat{\hat{\sigma}}^{1}\right)=\Theta \cdot f\left(\hat{\hat{\sigma}}^{1}\right)
$$

where

$$
\Theta \equiv \mathcal{B}_{(22)}^{\frac{1}{2}}\left(\frac{\mu}{\mu^{\prime}}\right)^{\frac{1}{2}}\left(\frac{|D|}{24 \pi}\right)^{-\frac{1}{2}}\left\{-\partial_{\hat{\hat{\sigma}}^{1}}^{2}+2 \exp (-2 \hat{\hat{\Phi}})+\frac{\hat{\hat{\sigma}}_{1}^{4}}{2} \exp (2 \hat{\hat{\Phi}})\right\} .
$$

Quantum perturbation theory at large $\boldsymbol{J}$. Prior to the rescaling executed above, it looked as though the theory contained any number of operators that could become singular at the boundary. We now see, however, that there is indeed a controlled perturbative expansion of the effective Lagrangian near the boundary in $\breve{\phi}$ propagators and vertices at large $J$, by using (3.12) and (3.13) in the action. Translational invariance near the boundary is strongly broken, so the Gaussian terms for the propagator are position-dependent, but, even so, their $J$-scaling is simple and can be read off directly from the Lagrangian for $\breve{\phi}$. That is, the Gaussian action has the form

$$
\mathcal{L}_{\text {Gaussian }}^{(\breve{\phi})}=\left(\frac{|D|}{24 \pi}\right)\left(\dot{\check{\phi}}^{2}+\breve{\phi} \Theta^{\text {(gauss) }} \breve{\phi}\right)
$$

where $\Theta^{\text {(gauss) }}$ is just the operator in (3.25). Therefore, the $\breve{\phi}$-propagator is always dominated by a position-dependent mass-squared term scaling as $\mathcal{B}_{(22)}^{1 / 2}$, which goes as $J^{\frac{1}{2}}$. Thus, the on-shell frequencies of near-boundary modes will always scale as $J^{1 / 4}$.

\subsection{From UV operators to effective boundary operators}

Now we would like to show that any gauge invariant boundary operator in the microscopic theory, or likewise any bulk operator in the scaling region $\hat{\hat{\sigma}}_{1}=O(1)$, goes over to an operator satisfying the $\mathcal{B}_{(22)}$-dressing rule on the boundary in the effective theory.

To begin, it is useful to write the full expansion of the bulk operator $\mathcal{I}_{11}$ near the boundary:

$$
\mathcal{I}_{11}=\sum_{j=2}^{\infty} \mathcal{O}_{11}^{(j)} \sigma_{1}^{j}=\sum_{j=2}^{\infty} \Xi^{j} \mathcal{O}_{11}^{(j)} \mathcal{B}_{(22)}^{-j / 4} \hat{\hat{\sigma}}_{1}^{j}
$$

where

$$
\left.\mathcal{O}_{k l}^{(j)} \equiv \frac{1}{j !} \partial_{\sigma^{1}}^{j} \mathcal{I}_{k l}\right|_{\sigma^{1}=0},
$$


and $\Xi$ is a numerical constant given by

$$
\Xi \equiv\left(\frac{|D|}{24 \pi}\right)^{\frac{1}{4}} \mu^{-\frac{1}{4}} \mu^{\prime \frac{1}{4}}
$$

The $\mathcal{O}_{k l}^{(j)}$ consist of operators of the form $\mathcal{B}_{(p q)}$ with $p+q=j+k+l$.

Now, we consider the most general monomial perturbation of the Lagrangian respecting Poincaré and worldsheet scale invariance:

$$
\mathcal{L}_{\text {pert }}=e^{M \phi} \prod_{p, q \geq 1} \mathcal{I}_{p q}^{N_{p q}} \prod_{r \geq 1}\left(\partial^{r} \phi\right)^{K_{r}},
$$

where $M, N_{p q}$ and $K_{r}$ are some arbitrary exponents. For this contribution to be of mass dimension two, we require

$$
-M+\sum_{p, q \geq 2}(p+q) N_{p q}+\sum_{r \geq 1} r K_{r}=2 .
$$

Using this restriction to eliminate $M$, let us rewrite $\mathcal{L}_{\text {pert }}$ as

$$
\mathcal{L}_{\text {pert }}=e^{-2 \phi} \prod_{p, q \geq 1} \mathcal{S}_{p q}^{N_{p q}} \prod_{r \geq 1} \mathcal{T}_{r}^{K_{r}}
$$

where

$$
\mathcal{S}_{p q} \equiv \exp \{(p+q) \phi\} \mathcal{I}_{p q}, \quad \mathcal{T}_{r} \equiv \exp (r \phi) \partial^{r} \phi .
$$

The objects $\mathcal{S}_{p q}$ and $\mathcal{T}_{r}$ have scaling dimension zero, so $\mathcal{L}_{\text {pert }}$ is now strictly of mass dimension two.

We can now compute constraints on the $|X|$-scaling of terms in $\mathcal{L}_{\text {pert }}$ in the boundary region. In terms of the shifted field $\hat{\hat{\phi}}(3.12)$,

$$
\exp \{(p+q) \phi\} \sim \mathcal{B}_{(22)}^{-(p+q) / 4} \exp \{(p+q) \hat{\hat{\phi}}\},
$$

so the $\mathcal{S}_{p q}$ contribution to the $|X|$-scaling of $\mathcal{L}_{\text {pert }}$ at the boundary is $2-(p+q) / 2$. Now, the only $\mathcal{S}_{p q}$ that can potentially contribute positive $|X|$-scaling overall are those for which $p+q \in\{2,3\} . \mathcal{I}_{11}$ scales at the boundary as $-\frac{1}{2} \mathcal{B}_{(22)} \sigma_{1}^{2}=-\frac{1}{2} \Xi^{2} \mathcal{B}_{(22)}^{1 / 2} \hat{\hat{\sigma}}_{1}^{2}$, so

$$
\mathcal{S}_{11}=\exp (2 \phi) \mathcal{I}_{11} \sim-\frac{\mu \mu^{\prime}}{2} \mathcal{B}_{(22)}^{0} \exp (2 \hat{\hat{\phi}}) \hat{\hat{\sigma}}_{1}^{2}=O\left(|X|^{0}\right) .
$$

Similarly, $\mathcal{I}_{12}$ and $\mathcal{I}_{21}$ at the boundary behave like $\pm \frac{1}{2} \mathcal{B}_{(22)} \sigma_{1} \sim \pm \frac{1}{2} \mathcal{B}_{(22)}^{3 / 4} \hat{\hat{\sigma}}_{1}$, so $\mathcal{S}_{12}$ and $\mathcal{S}_{21}$ go as $\pm \frac{1}{2} \mathcal{B}_{(22)}^{0} \exp (3 \hat{\hat{\phi}}) \hat{\hat{\sigma}}_{1}=O\left(|X|^{0}\right)$.

The $\mathcal{B}_{(22)}$ scaling of factors of the form $\mathcal{T}_{r}$ can be analyzed in a similar fashion. The $\mathcal{B}_{(22)}$-scaling of contributions from $\exp (r \phi)$ are $\mathcal{B}_{(22)}^{-r / 4}$, while the dominant $\mathcal{B}_{(22)}$ contributions from the $\phi$ derivatives in $\mathcal{T}_{r}$ descend from $\partial_{1}$ derivatives, and these become $\partial_{1}^{r}=\Xi^{-r} \mathcal{B}_{(22)}^{r / 4} \partial_{\hat{\sigma}^{1}}^{r}$ in our rescaled coordinates. So the $\mathcal{T}_{r}$ objects themselves enter with $\mathcal{B}_{(22)}$-scaling $\mathcal{B}_{(22)}^{0}$. Thus, we have seen that the $\mathcal{B}_{(22)}$-dressing rule is satisfied in the effective theory, for any operator insertion in the UV theory of the general form (3.30). 
As a specific example, we now demonstrate that the boundary Liouville term in the microscopic theory descends to a quark mass term in the effective string theory. In this regime, we can calculate the numerical value of the coefficient of the quark mass operator in the effective string theory derived from the Polchinski-Strominger deformed Liouville theory, with space-filling branes in $D+1$ dimensions, and a boundary Liouville term:

$$
\mathcal{L}_{\text {boundary }}=\mu_{B} \exp (-\Phi) \rightarrow \frac{0.6558 \mu_{B}}{\left(\mu^{3} \mu^{\prime}\right)^{\frac{1}{4}}}\left(\frac{|D|}{24 \pi}\right)^{\frac{1}{4}} \mathcal{B}_{(22)}^{\frac{1}{4}} .
$$

When we expand the $X$ field in vev plus fluctuations, the terms with fluctuations have lower $J$-scaling than the $\mathcal{B}_{(22)}$ term evaluated in the classical solution. Therefore, the coefficient of the $J^{1 / 4}$ term in the open string mass-squared is set directly by the coefficient in equation (3.36), regardless of the details of the state, so long as its excitation number above the ground state is not parametrically large in $J$.

\subsection{Quantum corrections leave the boundary dressing rule unmodified}

To show that quantum corrections do not affect the dressing rule at the nonperturbative level, all we need to establish is that the energies of the modes we are integrating out go as $\omega_{\sigma^{0}} \sim \mathcal{B}_{(22)}^{1 / 4}$. In a Wilsonian action, the dimensional suppression of a nonrenormalizable effective term is generated by inverse powers of the frequencies of the modes that generated the term when they were integrated out. Bulk terms are generated by integrating out bulk modes of the Liouville field, whose bulk mass is proportional to $\sqrt{\left|\mathcal{I}_{11}\right|}$. On the other hand, near the boundary, the dimensional suppression of boundary operators comes from negative powers of the frequency of near-boundary modes of the fluctuations of $\phi$. From equation (3.22) we can see that the frequencies of these modes are of order

$$
\omega=O\left(\mathcal{B}_{(22)}^{\frac{1}{4}}\left(\frac{|D|}{24 \pi}\right)^{-\frac{1}{4}}\right) .
$$

It follows that $\mathcal{B}_{(22)}$ is indeed the operator dressing for all boundary operators. This is a nonperturbative statement, and the powers of $\omega$ that appear - and therefore the powers of $\mathcal{B}_{(22)}$ that appear - depend on the full nonperturbative dynamics of the strongly coupled conformal field theory through the anomalous dimensions of the operators they are dressing. Note, however, that the form of the dressing, as opposed to its exponents, is the same as it is in large- $|D|$ perturbation theory: both the classical solution for $\phi$ and the propagator for its fluctuations, contain only powers and logarithms of $\mathcal{B}_{(22)}$.

\section{A displaced-boundary regulator}

To this point, we have been working from the starting point of a UV-complete worldsheet theory, taking the form of a Liouville model coupled to the goldstone bosons $X$. As we have seen, this ultraviolet completion provides a natural way to regulate the effective string theory, giving the $\mathcal{B}_{(22)}$-dressing rule for effective Neumann boundary operators, as well as the $\mathcal{I}_{11}$-dressing rule for bulk effective operators. Deriving the dressing rule from a UVcomplete theory, however, should not overshadow one very important point: the structure 
of these operator dressing rules is an intrinsic property of the effective theory. That is, the operator dressing rules we have demonstrated above do not arise as an artifact of a particular UV-completion of effective string theory. To illustrate this point in greater detail, we can adopt a different regulation procedure that is not related to any particular physical completion of the effective theory. For instance, we can work instead with an artificial cutoff, which renders calculations tractable while preserving the underlying symmetries of the worldsheet theory. The $\mathcal{B}_{(22)}$-dressing rule again emerges ineluctably in such schemes, so long as one takes care to preserve worldsheet gauge symmetries, ${ }^{7}$ as well as the global $D$-dimensional Poincaré symmetry.

One such regulator is defined by replacing the boundary of the worldsheet with a displaced timelike boundary, moved slightly into the interior of the worldsheet by a fixed distance $\epsilon$. We refer to this scheme as the "displaced-boundary regulator." For bulk operators, the significance of the displaced boundary is that we integrate only up to the displaced boundary rather than the real one, excising interaction terms from the strip at the boundary. For boundary operators, we integrate along the displaced boundary rather than the real one. For instance, for a quark mass, we simply let the worldline of the quark run along the displaced boundary.

The spacelike displaement $\epsilon$ is measured with respect to the induced metric. This regulator is fully gauge invariant by construction, referring only to gauge invariant quantities, and approaches the bare quark action when $\epsilon$ is taken to zero.

To avoid complications in the near-boundary expansion, we can define the induced proper distance with respect to the free, rather than interacting, $X$-coordinates, which is indeed equivalent to turning off all bulk interactions inside the excised strip. This is a perfectly well-defined and gauge-invariant procedure, modulo one subtle complication: when we work in the simplified covariant formalism, we need the anomaly term to maintain gauge invariance at the quantum level. Therefore turning off the anomaly term in the nearboundary strip is not a completely legitimate operation. However, the only effect of the excision of the anomaly term is a variation of the quantum effective action by the WessZumino functional integrated over a strip of coordinate width proportional to $\epsilon^{\frac{1}{2}}$. This goes to zero as $\epsilon \rightarrow 0$, and gauge invariance is restored as the regulator is removed. Concretely, we will see below that the effect of the near-boundary excision of the anomaly term is equivalent to adding a gauge-invariant boundary counterterm, plus other boundary terms that vanish in the limit $\epsilon \rightarrow 0$.

Alternatively, we can define the induced proper distance with respect to the interacting embedding coordinates. If we do so, we must separately regulate the bulk interactions (including the anomaly term) and remove the associated divergences with boundary counterterms again. $^{8}$

\footnotetext{
${ }^{7}$ In this paper we work within the framework of the simplified covariant formalism for the bosonic string [3], which is based on the Polyakov formalism with the addition of an anomaly-cancelling term. In this formalism the gauge symmetries are worldsheet diffeomorphisms and Weyl transformations.

${ }^{8}$ We give an example of a regulator of this type in appendix A, where we perform a fully gauge-invariant calculation of the asymptotic intercept. In this calculation, the excision of the strip is not needed, as the only divergence comes from the bulk anomaly term, which we regulate explicitly.
} 
In what follows, we work through three examples of interest, and the displacedboundary regulator proposed here handles each differently. First, we consider boundary terms. The naive quark-mass operator, regulated according to this scheme, requires a multiplicative renormalization in order to have nonzero matrix elements in low-energy states in the limit where the regulator is removed. It is important to note that this is always true when we are expanding in the limit where the quark mass is held fixed, at any finite value, and $J$ is taken to infinity. Other boundary operators, such as the integrated geodesic curvature, or the proper acceleration operator, are proportional to the quark-mass operator at leading order in $J$.

Second, we investigate fully gauge invariant bulk terms. For one such example, that of the induced-curvature-squared term, we compute the leading divergence as the size of the displaced-boundary regulator is taken to zero. Here again, the divergence is proportional to the quark mass term, with a coefficient scaling as $\epsilon^{-5 / 2}$.

Finally, we consider the anomaly term itself, which we need to include when working in the Polyakov formalism, in order to maintain gauge invariance at the quantum level $[2,3]$. The naive near-boundary regulation of this term does not result in a gauge-invariant theory, because the anomalous transformation of the free theory is cancelled only by the integral of the PS term over the full worldsheet. In section 4.4, we show that gauge invariance is restored in the limit $\epsilon \rightarrow 0$, by decomposing the integral of the PS term in the strip as a gauge invariant operator (proportional to $\mathcal{B}_{(22)}^{1 / 4}$ ) and terms that vanish as $\epsilon \rightarrow 0$.

More generally, and returning to the central lesson of the dressing rule, there is only one invariant perturbation of the action of order $J^{1 / 4}$, and all gauge-invariant operators are just proportional to a single, linearly independent operator, $\mathcal{B}_{(22)}^{1 / 4}$, at order $J^{1 / 4}$. This operator should be thought of as just the identity, dressed with a line element along the boundary, expanded near the boundary and renormalized multiplicatively to give a finite and nonzero value.

\subsection{Definition of the displaced-boundary regulator scheme}

To begin, we attach the worldline of the quark to the string worldsheet, separated from the boundary by a fixed spacelike induced proper distance $\epsilon$. That is, the distance from the quark to the boundary is computed with respect to the induced metric on the worldsheet,

$$
G_{\sigma^{a} \sigma^{b}}^{(\text {ind })} \equiv \partial_{a} X \cdot \partial_{b} X
$$

The simplest gauge-invariant characterization of a trajectory near the boundary is defined to be the set of interior points separated from the boundary by extremal spacelike geodesics of length $\epsilon$ with respect to the induced metric. ${ }^{9}$ The spacelike geodesics of interest connecting interior points to the boundary are simply slices of constant $\sigma^{0}$, parametrized by $\sigma^{1}$. That is, the set of points lying at a fixed spacelike geodesic distance $\epsilon$ from the boundary

\footnotetext{
${ }^{9}$ In particular, the separation is characterized by spacelike geodesics of maximal length (in Lorentzian signature), extending from an interior reference point to the boundary. See appendix B for further details on the maximal geodesic in the near-boundary region.
} 
is just a trajectory of constant $\sigma_{1}$ :

$$
\sigma_{1}=\tilde{\sigma}=\left(\sigma^{0}-\text { independent }\right) \text {. }
$$

With this, we can work out the actual value of the coordinate location $\tilde{\sigma}$ of the nearboundary trajectory, in terms of the induced proper distance $\epsilon$.

Concretely, the induced proper distance is

$$
\epsilon=\int_{0}^{\tilde{\sigma}} d \sigma \sqrt{G_{\sigma \sigma}^{(\mathrm{ind})}}=\int_{0}^{\tilde{\sigma}} d \sigma \sqrt{\partial_{\sigma} X^{\mu} \partial_{\sigma} X_{\mu}}=\int_{0}^{\tilde{\sigma}} d \sigma \sqrt{-2 \mathcal{I}_{11}} .
$$

Recalling the boundary expansion of $\mathcal{I}_{11}$ in eq. (3.11), we obtain

$$
\epsilon=\int_{0}^{\tilde{\sigma}} d \sigma \sqrt{\mathcal{B}_{(22)} \sigma^{2}+O\left(\sigma^{3}\right)}=\frac{1}{2} \sqrt{\mathcal{B}_{(22)}} \tilde{\sigma}^{2}+O\left(\tilde{\sigma}^{3}\right)
$$

or

$$
\tilde{\sigma}=\sqrt{2 \epsilon} \mathcal{B}_{(22)}^{-\frac{1}{4}}+O(\epsilon) .
$$

The expressions in this section are again operator identities, and not simply properties of the classical helical solution; this point warrants some discussion. The expansion of $\mathcal{I}_{11}$ near the boundary in (4.4) receives corrections due to bulk interactions, just as it did in the Polchinski-Strominger-Liouville UV completion, as discussed above (3.11). In the UV-complete theory, the bulk interactions were nonsingular near the boundary, and their near-boundary expansion was unproblematic. Strictly within the effective theory, however, we have bulk operators that are explicitly singular near the boundary, where these singularities are excised by the explicit cutoff at fixed induced proper length $\epsilon$ from the boundary.

For instance, there are terms coming from the PS correction and from other corrections to the free-field action and its constraints. The d'Alembertian on $X^{\mu}$ contains singular terms such as $\beta \alpha^{\prime} \frac{\mathcal{I}_{21} \mathcal{I}_{12}^{2}}{\mathcal{I}_{11}^{4}} \partial_{+} X^{\mu}$, leading to corrections to the r.h.s. of (3.11) of the form $\beta \alpha^{\prime} \frac{\mathcal{I}_{21}^{2} \mathcal{I}_{12}^{2}}{\mathcal{I}_{11}^{4}} \sigma_{1}^{2}$, for example, which, near the boundary, behave as

$$
\left.\beta \alpha^{\prime} \frac{\mathcal{I}_{21}^{2} \mathcal{I}_{12}^{2}}{\mathcal{I}_{11}^{4}} \sigma_{1}^{2}\right|_{\text {boundary }} \rightarrow \frac{\beta \alpha^{\prime}}{\sigma_{1}^{2}} .
$$

As noted above, we can deal with such singularities either by introducing a separate UV cutoff for the bulk interactions, or by simply defining the induced geodesic distance with respect to an embedding in which the embedding coordinates satisfy the free, rather than interacting, EOM inside the excised strip. We take the latter approach in this section.

It is also important to note that, while $\epsilon$ is a number, $\tilde{\sigma}\left(\sigma^{0}\right)$ is actually an operator, denoting the coordinate position at which the induced proper distance from the boundary is equal to $\epsilon$. The $\epsilon$-dependent terms coming from the regulator enter in a series with hierarchical $J$-suppression, determined by how many powers of the dressing operator $\mathcal{B}_{(22)}$ they carry in the denominator. 
There is a second kind of large- $J$ suppression associated with the expansion of any given operator into a classical background and quantum fluctuations, i.e.,

$$
X^{\mu} \equiv Y^{\mu}+E_{\text {helical }}^{\mu}
$$

where $E_{\text {helical }}^{\mu}$ is the helical classical solution, whose invariants (such as $\mathcal{I}_{11}$ ) are timeindependent. For any given configuration in the path integral, the length of the extremal geodesic integrated out to $\sigma_{1}=\tilde{\sigma}$ in the boundary scaling region contains corrections such as $\frac{1}{2} \sqrt{B_{(22)}} \tilde{\sigma}^{p} \mathcal{O}_{p}$, where $\mathcal{O}_{p}$ is an operator of dimension $p$ made of derivatives of $Y$, e.g., $\mathcal{O}_{p} \ni(\partial Y)^{p}$. Since $\tilde{\sigma}$ depends on the configuration and $\epsilon$ does not, it is better to express the corrections to (4.5) in terms of fixed- $\epsilon$ quantities,

$$
\tilde{\sigma}=\sqrt{2 \epsilon} \mathcal{B}_{(22)}^{-\frac{1}{4}}\left(1+O\left(\epsilon^{p / 2} B_{(22)}^{-p / 4} \mathcal{O}_{p}\right)\right) .
$$

Note, in particular, that the correction terms in the equation above will be suppressed both by $J$ and $\epsilon$.

\subsection{Boundary operators}

We now expand some simple gauge-invariant operators near the boundary, with the boundary proximity defined in a gauge-invariant way, based on the induced proper distance. The key result here is that gauge invariant local operators have near-boundary expansions that go as (const.) $J^{0}$, with the constant depending only on the regulator and the Hamiltonian of the system, and not at all on the state. In other words, the leading coefficient can be theory-dependent, but not state-dependent within a given effective theory. After being multiplied by the induced boundary line element to make a gauge-invariant perturbation to the action, gauge-invariant terms scale as (const.) $\mathcal{B}_{(22)}^{1 / 4}$, where the constant factor is independent of the state.

Quark mass operator from naive quark action. The simplest case to consider is the operator $\mathcal{B}_{(22)}$, in which we simply take the identity, multiply it by a regulated induced line element with a coefficient we can think of as a bare quark mass term. The naive quark mass term thus appears as

$$
S_{\text {(quark mass) }}=M^{(\text {bare })} \int d s^{(\text {induced })}=M \int d \rho \sqrt{-\frac{d X^{\mu}}{d \rho} \frac{d X_{\mu}}{d \rho}},
$$

where the integral is now understood to be taken over a timelike trajectory near the boundary, and where the separation from the boundary is parametrized by the cutoff $\epsilon$. Taking the integral over an exactly lightlike boundary leads to a singular worldsheet Hamiltonian; the nonsingular operator is obtained by rescaling the naive term. Stated another way, after fixing a gauge-invariant scheme parametrized by $\epsilon$ to regularize the term, we make an $\epsilon$-dependent readjustment of the bare parameter $M$ :

$$
S_{(\text {quark mass })}=M^{(\text {bare })}(\epsilon) \int d s^{\text {(induced) }} .
$$


As noted, the renormalization can depend on the scheme, and on the parameters of the worldsheet Hamiltonian, but cannot depend on the state of the system. Therefore, it is simplest to determine the scaling by simply inserting the quark mass term into the helical solution. Evaluated on this trajectory, the line element in (4.9) goes as

$$
\begin{aligned}
d \rho \sqrt{-\frac{d X^{\mu}}{d \rho} \frac{d X_{\mu}}{d \rho}} & =d \tau \sqrt{-\dot{X}^{2}}=d \tau \sqrt{-4 \mathcal{I}_{11}} \\
& \simeq d \tau \sqrt{2 \mathcal{B}_{(22)} \tilde{\sigma}^{2}}=2 d \tau \epsilon^{\frac{1}{2}} \mathcal{B}_{(22)}^{\frac{1}{4}},
\end{aligned}
$$

where we have used the Virasoro constraints to substitute $-\dot{X}^{2} \sim-4 \mathcal{I}_{11}$, and equation (3.11) to approximate $\mathcal{I}_{11}$ by $-\frac{1}{2} \mathcal{B}_{(22)} \tilde{\sigma}^{2}$. Thus, the bare mass $M^{(\text {bare })}(\epsilon)$ must be scaled as $\epsilon^{-\frac{1}{2}}$ to recover a finite operator when $\epsilon$ is taken to 0 :

$$
M^{(\text {bare })}(\epsilon) \sim c_{\text {quark }} \epsilon^{-\frac{1}{2}} .
$$

We have discarded subleading terms on the r.h.s. of (4.11) such as $\epsilon \mathcal{B}_{(23)} / \mathcal{B}_{(22)}^{3 / 2}$. In general, boundary operators contributing to $\tilde{\sigma}$ have scaling dimension -1 and so must come dressed with an $\left(\epsilon^{2} / \mathcal{B}_{(22)}\right)^{\frac{p+q-4}{4}}$ for every $\mathcal{B}_{(p q)}$ in the numerator. The expansion of the formula for $\tilde{\sigma}$ in boundary operators is therefore an expansion in (fractional) positive powers of $\epsilon^{2} / J \alpha^{\prime}$.

Note that all throughout, we have taken the physical effective quark mass term, defined as the $J^{1 / 4}$ contribution to the mass-squared, to be zero prior to the perturbation (4.9). In the presence of a nonzero quark mass coefficient, the boundary of the worldsheet is slightly timelike at finite $J$, and the operator renormalization (4.12) may be deformed. ${ }^{10}$ However, the basic organization of boundary operators itself does not depend on the value of $c_{\text {quark }}$ : the basis of boundary operators is still the set of arbitrary polynomials of $X$ and its derivatives, dressed with powers of $\mathcal{B}_{(22)}$, so long as $c_{\text {quark }}$ is taken fixed and independent of $J$ as $J$ is taken to infinity. ${ }^{11}$

Boundary operator: geodesic curvature. Another example of a boundary operator is the geodesic curvature. Taking $\gamma$ to be a curve of fixed $\sigma$, with unit tangent vector $u=d \gamma / d s$, we have

$$
\begin{aligned}
u & =\frac{d \gamma}{d s}=\frac{\left(\tau^{\prime}(\rho), \sigma^{\prime}(\rho)\right)}{\left|g_{a b} \sigma^{a \prime}(\rho) \sigma^{b \prime}(\rho)\right|^{1 / 2}} \\
& =\frac{(1,0)}{\sqrt{2\left|\mathcal{I}_{11}\right|}},
\end{aligned}
$$

where we have chosen the specific parameterization $\tau=\rho$. The geodesic curvature squared is

$$
\kappa^{2}=u^{c} u^{d} D_{c} u^{a} D_{d} u^{b} g_{a b} .
$$

\footnotetext{
${ }^{10}$ We thank S. Dubovsky and V. Gorbenko for discussions of this point.

${ }^{11}$ Note that it is also possible to take a scaling limit where the coefficient of the $\mathcal{B}_{(22)}^{\frac{1}{4}}$ term is scaled as $J^{\frac{3}{4}}$, in which the velocity of the endpoint stays finite and the organization of boundary operators changes. See, for instance, the discussion of rotating strings at large $J$ and fixed endpoint velocity in [14].
} 
Expressing this quantity in terms of the embedding coordinates via the induced metric, we obtain

$$
\kappa^{2}=\frac{1}{4}\left(u^{0}\right)^{4} g^{11}\left(g_{00,1}\right)^{2}=\frac{\left(\dot{X} \cdot \dot{X}^{\prime}\right)^{2}}{(\dot{X} \cdot \dot{X})^{3}} .
$$

Taken to the boundary cutoff $\epsilon$,

$$
\kappa^{2}=\left.\frac{1}{\mathcal{B}_{(22)} \sigma^{4}}\right|_{\epsilon}
$$

where $\tilde{\sigma}=\sqrt{2 \epsilon} \mathcal{B}_{(22)}^{-1 / 4}+O(\epsilon)$, we obtain

$$
\kappa^{2} \longrightarrow \frac{1}{4 \epsilon^{2}}
$$

The matrix elements of this operator are theory-dependent, regulator-dependent, and UV singular for massless endpoints, but have the same value at order $J^{0}$ for every state in the low-energy Hilbert space. Treated as integrated perturbations, they scale as $J^{1 / 4}$ after multiplication by the induced boundary line element $\mathcal{B}_{(22)}^{1 / 4}$.

Boundary operator: proper acceleration. Let us now consider the proper acceleration,

$$
a^{a}=X_{, \rho \rho}^{a}+\Gamma_{b c}^{a} X_{, \rho}^{b} X_{, \rho}^{c},
$$

where it can be shown by similar methods that, near the boundary,

$$
a_{\rho \rho}^{a} a_{\rho \rho}^{a}\left(g^{\rho \rho}\right)^{2}=\frac{(\ddot{X})^{2}}{\left(\dot{X}^{2}\right)^{2}}=\frac{\mathcal{B}_{(22)}}{\left(\mathcal{B}_{(22)} \tilde{\sigma}^{2}\right)^{2}}=\frac{1}{4 \epsilon^{2}} .
$$

We have now expanded several gauge-invariant operators in a near-boundary expansion, where the distance to the boundary is defined in a gauge-invariant way. With the regulation parameter $\epsilon$ held fixed, we have found that gauge-invariant scalar operators scale as $J^{0}$ in the large- $J$ limit, with a coefficient that depends only on the parameters of the theory and on the regulator, and not on the individual state of the system. In particular, the coefficient can be read off directly from its value in the helical state. After multiplication by the regulated line element, which scales as $\mathcal{B}_{(22)}^{1 / 4}$, these operators contribute to the action at leading order as $J^{1 / 4}$, with a state-independent coefficient. The operators in the examples above demonstrate the dressing rule concretely, in the context of a particular short-distance cutoff.

\subsection{Bulk operators}

As an example of a bulk operator analysis in the displaced-boundary regulator scheme, consider the curvature-squared of the induced metric:

$$
\tilde{R}^{2} d \mu_{\text {Induced }}=8 \frac{\left(\hat{\mathcal{I}}_{22}\right)^{2}}{\left|\mathcal{I}_{11}\right|^{3}} d \sigma^{0} d \sigma^{1}
$$


where, as in [3], we define $\widehat{\mathcal{I}}_{22}$ to be the Weyl-covariant version of $\mathcal{I}_{22}$ :

$$
\widehat{\mathcal{I}}_{22} \equiv \mathcal{I}_{22}-\frac{\mathcal{I}_{12} \mathcal{I}_{21}}{\mathcal{I}_{11}}
$$

Near the boundary, this becomes

$$
\left.\frac{\left(\widehat{\mathcal{I}}_{22}\right)^{2}}{\mathcal{I}_{11}^{3}}\right|_{\text {bdy }}=-\frac{1}{2} \mathcal{B}_{(22)}^{-1} \sigma^{-6},
$$

so that

$$
\begin{aligned}
\int_{\tilde{\sigma}}^{\sigma_{\mathrm{far}}} \tilde{R}^{2} d \sigma & =\int_{\tilde{\sigma}}^{\sigma_{\text {far }}} 8 \frac{\left(\widehat{\mathcal{I}}_{22}\right)^{2}}{\mathcal{I}_{11}^{3}} d \sigma \\
& =\frac{4}{5} \mathcal{B}_{(22)}^{-1}\left(\sigma_{\text {far }}^{-5}-\tilde{\sigma}^{-5}\right) \\
& =\frac{4}{5} \mathcal{B}_{(22)}^{-1}\left(\sigma_{\text {far }}^{-5}-\frac{1}{(2 \epsilon)^{5 / 2} \mathcal{B}_{(22)}^{-5 / 4}}\right) \\
& =-\frac{4}{5}(2 \epsilon)^{-\frac{5}{2}} \mathcal{B}_{(22)}^{\frac{1}{4}} .
\end{aligned}
$$

Note that we have dropped subleading divergent terms in the near-boundary expansion, which give rise to operators of lower $X$-scaling and less divergent $\epsilon$-scaling than the leading term (4.23). For instance, including the $\sigma^{-4}$ term in the near-boundary expansion of $\mathcal{I}_{11}^{-3}$ leads to a divergence proportional to $\mathcal{B}_{(33)} / \mathcal{B}_{(22)}^{5 / 4} \epsilon^{-3 / 2}$ in the integrated curvature-squared term, which scales as $J^{-1 / 4}$. In addition, there are similar corrections coming from the subleading terms in the expansion of $\tilde{\sigma}$ in powers of $\epsilon^{2} / J \alpha^{\prime}$, discussed below (4.5).

In particular, any $\epsilon$-independent terms in the integral must scale as $1 /\left(J \alpha^{\prime}\right)$ at most, simply by dimensional analysis. Thus, while the UV-divergent counterterms may have $J$ scaling as large as $J^{1 / 4}$, the observable, finite contribution of the curvature-squared operator to the mass-squared of the open string state is no greater than $O\left(J^{-1}\right)$. More generally, a higher-derivative bulk operator with $|X|$-scaling $|X|^{-p}$ may generate UV divergences going as $\epsilon^{p^{\prime}-p} \mathcal{O}_{\left(p^{\prime}\right)}$, where $\mathcal{O}_{\left(p^{\prime}\right)}$ is an operator with $|X|$-scaling $-p^{\prime}$. Finite terms can only scale as $O\left(J^{-p / 2}\right)$ at most. This is the basis for the perturbativity of the properly renormalized effective worldsheet theory in the $1 / J$ expansion.

\subsection{Anomaly term}

The displaced-boundary regulator treatment of the anomaly term must be handled somewhat more cautiously than the corresponding regulation of gauge-invariant bulk terms. The naive regulation of the anomaly term does not result in a gauge-invariant path integral, because the anomalous transformation of the free theory is cancelled only by the integral of the PS term over the full worldsheet.

One way to proceed is to use a modified PS term that is finite at the boundary while preserving exact gauge invariance. Such a term can be constructed easily in the general framework of [3]. This approach yields a notably different situation, with two classes 
of bulk terms. One class admits gauge-invariant terms of definite $X$-scaling, which are integrated over the worldsheet with the boundary strip removed. The other class of bulk terms have inhomogeneous $X$-scaling by necessity. They behave like a Liouville term under Weyl transformations, and are integrated over the entire strip.

Another approach is simply to integrate the standard, unmodified anomaly term over the worldsheet with the excised strip, and notice that the only term with a nonpositive $\epsilon$-scaling is the quark mass term, which is gauge invariant. Modulo terms that vanish as $\epsilon \rightarrow 0$, the strip-excision prescription for the anomaly term results here in a gauge-invariant and finite quantum effective action (because one can renormalize the sole divergence with a gauge-invariant counterterm).

Near the boundary, it can be shown that

$$
\mathcal{L}_{\mathrm{PS}}=-\frac{\beta}{2 \pi} \frac{1}{\sigma^{2}}+O\left(\sigma^{0}\right) .
$$

The displaced-boundary regulator integral then yields

$$
\int_{\tilde{\sigma}}^{\sigma_{\mathrm{far}}} \mathcal{L}_{\mathrm{PS}} d \sigma=-\frac{\beta}{2 \pi} \frac{\mathcal{B}_{(22)}^{1 / 4}}{\sqrt{2 \epsilon}}+O\left(\epsilon^{0}\right) .
$$

This defines the counterterm to be added to the action. Although this regulator does not, strictly speaking, preserve gauge invariance for $\epsilon \neq 0$, we proceed by adding

$$
\Delta \mathcal{L}=\left(c_{\text {divergent }}+c_{\text {finite }}\right) \mathcal{B}_{(22)}^{1 / 4},
$$

with

$$
c_{\text {divergent }}=\frac{\beta}{2 \pi} \frac{1}{\sqrt{2 \epsilon}} .
$$

We are free to choose $c_{\text {finite }}$, as long as it is $\epsilon$-independent. The result is a gauge invariant, finite path integral in the limit $\epsilon \rightarrow 0$. This concretely illustrates the restoration of gauge invariance in the limit $\epsilon \rightarrow 0$ : the near-boundary region's contribution to the anomaly action is seen explicitly to be equal to a (gauge-invariant) boundary term, plus contributions scaling as positive powers of $\epsilon$.

\section{Conclusions and physical consequences}

We have proposed an operator dressing rule for Neumann boundaries in effective string theory in which the boundary operator $\mathcal{B}_{(22)}$ plays the role of the unique monomial in $X$ occurring to negative or fractional powers, analogous to $\mathcal{I}_{11}$ for bulk operators. Having motivated the rule via a number of heuristic arguments, we then explicitly derived the $\mathcal{B}_{(22)}$ dressing rule for Neumann boundaries in detail in a particular UV completion of the effective theory. To avoid any reliance on the specific UV completion, we also demonstrated the persistence of the dressing rule using an artificial regulator directly in the effective theory.

There is at least one important physical consequence of this analysis. Having established the dressing rule for Neumann boundaries, we can explain in detail the universality 
of the asymptotic Regge intercept for mesons in planar QCD with massless, bosonic quarks, first calculated in [4]. (For completeness, we include an explicit, gauge-invariant calculation of the asymptotic Regge intercept in this theory - for bosonic quarks on the leading trajectory, in the planar approximation - in appendix A.) The argument stems from the fact that all bilinear invariants of the embedding coordinates $X$ at the boundary are of the form

$$
\mathcal{B}_{(p q)} \equiv \partial_{0}^{p} X \cdot \partial_{0}^{q} X
$$

and, by the dressing rule, boundary operators are spanned by the set

$$
\prod_{i} \mathcal{B}_{\left(p_{i} q_{i}\right)} / \mathcal{B}_{(22)}^{k}
$$

Now, consider only boundary operators of marginal scaling dimension. If an "undressed" operator (the numerator) has dimension

$$
\Delta \equiv \sum_{i} p_{i}+q_{i}
$$

then the dressing, under the requirement of conformality, is

$$
\mathcal{B}_{(22)}^{-(\Delta-1) / 4} .
$$

Thus, to have positive or zero $X$-scaling, the undressed operator must have $\Delta \leq 5$. The operators $\mathcal{B}_{(11)}$ and $\mathcal{B}_{(12)}$, for instance, vanish as independent operators because they are proportional to free-field stress tensors and first derivatives thereof. Meanwhile, the only marginal operator with $\Delta=5$ is $\mathcal{B}_{(23)} / \mathcal{B}_{(22)}$, which is a total derivative along the boundary. Thus, after modding out by Virasoro descendants, the only marginal operator with nonnegative $X$-scaling is the quark mass operator, corresponding to $\Delta=4$. There are no operators scaling as $J^{0}$, so the $J^{0}$ term in the expansion of the quantum effective action is indeed universal. In particular, the order $J^{0}$ term in the expansion of the mass-squared of the meson is independent of the details of the theory, beyond the basic assumptions of $D$-dimensional Poincaré invariance and the restriction that the Nambu-Goldstone bosons constitute the only infinite-range excitations on the string worldvolume.

It is also worth revisiting what we expect to hold as corresponding dressing rules when Dirichlet boundaries are included. In the case of strictly Dirichlet boundary conditions, the dressing rule should be formulated purely in powers of $\left(\dot{X}^{\prime}\right)^{2}$. When both Neumann and Dirichlet directions are present, we expect the appropriate dressing rule to be formulated in terms of $\left(\dot{X}_{\text {NeUMANN }}\right)^{2}$, or, equivalently by virtue of Virasoro constraints, in terms of $\left(X_{\text {DiRichleT }}^{\prime}\right)^{2}$.

Looking ahead, the renormalization analysis in this paper can and should be extended to the fold singularities of rotating strings with angular momentum in a single plane (see [3, 4, 15-17] and references therein for further discussion on this topic). It would also be interesting to understand the origin of the Neumann dressing rule in the context of Natsuume's warped UV completion [18], as this ties in most directly with modern holographic ideas in this arena. 


\section{A Calculation of the asymptotic Regge intercept}

In previous work [4] we presented an abridged computation of the first sub-leading correction near large $J$ to the ground state energy of spinning strings, arising from contributions from the Casimir energy and from the Polchinski-Strominger anomaly term discussed above. (The origin of the PS term in the general setting of a perturbed Liouville theory embedded in the Polyakov framework was further explained in [3].) In this section we provide an explicit and completely gauge-invariant derivation of these universal sub-leading corrections to the energy spectrum for open strings.

From [3, 4], the ground state helical solution discussed above can be explicitly written as

$$
\begin{aligned}
& X^{0}=2 \alpha^{\prime} P^{0} \sigma^{0} \\
& \bar{Z}_{1}=i \sqrt{\frac{\alpha^{\prime}}{2}} \alpha_{1}^{\bar{Z}_{1}}\left(e^{-i \sigma^{+}}+e^{-i \sigma^{-}}\right) \\
& \bar{Z}_{2}=i \sqrt{\frac{\alpha^{\prime}}{2} \frac{\alpha_{2}^{Z_{2}}}{2}\left(e^{-2 i \sigma^{+}}+e^{-2 i \sigma^{-}}\right)} \\
& Z_{1}=-i \sqrt{\frac{\alpha^{\prime}}{2}} \alpha_{-1}^{Z_{1}}\left(e^{i \sigma^{+}}+e^{i \sigma^{-}}\right) \\
& Z_{2}=-i \sqrt{\frac{\alpha^{\prime}}{2}} \frac{\alpha_{-2}^{Z_{2}}}{2}\left(e^{2 i \sigma^{+}}+e^{2 i \sigma^{-}}\right),
\end{aligned}
$$

with

$$
\begin{array}{ll}
\alpha_{1}^{\bar{Z}_{1}}=\sqrt{2 J_{1}} & \alpha_{-1}^{Z_{1}}=\sqrt{2 J_{1}} \\
\alpha_{2}^{\bar{Z}_{2}}=2 \sqrt{J_{2}} & \alpha_{-2}^{Z_{2}}=2 \sqrt{J_{2}} .
\end{array}
$$

The usual classical constraint takes the form,

$$
T_{++}=-\left(\partial_{+} X^{0}\right)^{2}+\partial_{+} Z_{1} \partial_{+} \bar{Z}_{1}+\partial_{+} Z_{2} \partial_{+} \bar{Z}_{2}
$$

which sets

$$
\left(P^{0}\right)^{2}=\frac{J_{1}+2 J_{2}}{\alpha^{\prime}}
$$

In $D \geq 5$, spinning strings can carry angular momenta $J_{1,2}$ in one or two planes, and the large- $J$ perturbation theory is understood to keep these quantities in fixed ratio. As described in [4], in a suitable Cartan decomposition, the angular momenta are aligned with the "3" direction of the self-dual and antiself-dual $\mathrm{SU}(2)_{ \pm}$subgroups of the $\mathrm{SO}(4)$ little group of $\mathrm{SO}(D-1)$. In $D \geq 5$, states can carry angular momenta in both planes with angular-momentum quantum numbers

$$
J_{ \pm}=\frac{1}{2}\left(J_{1} \pm J_{2}\right)
$$


States are determined by minimizing the energy over highest-weight vectors of $\mathrm{SU}(2)_{+} \times$ $\mathrm{SU}(2)_{-}$, with total angular momenta $J_{ \pm}$and zero momentum in the $\sigma^{1}$ direction. The free-field ground state in the open-string sector is unique and can be expressed as

$$
\left|J_{+}, J_{-} ; P\right\rangle_{\text {free }}=\frac{1}{\sqrt{\mathcal{N}_{J_{+}, J_{-}}^{\text {(open) }}}}\left(\alpha_{-1}^{Z_{1}} \alpha_{-2}^{Z_{2}}-\alpha_{-2}^{Z_{1}} \alpha_{-1}^{Z_{2}}\right)^{J_{+}-J_{-}}\left(\alpha_{-1}^{Z_{1}}\right)^{2 J_{-}}|0 ; P\rangle_{\text {free }} \text {. }
$$

The quantity $\mathcal{N}_{J_{+}, J_{-}}^{(\text {open })}$ is a normalization constant, and the energy under the free-field Hamiltonian takes the form

$$
E^{(\text {free })}=\alpha^{\prime} P^{2}+3 J_{+}-J_{-}-\frac{D}{24} .
$$

Starting with $\widehat{\mathcal{I}}_{22}$ in eq. (4.21) above,

$$
\widehat{\mathcal{I}}_{22} \equiv \mathcal{I}_{22}-\frac{\mathcal{I}_{12} \mathcal{I}_{21}}{\mathcal{I}_{11}}
$$

and adopting notation consistent with [3], we introduce a regulated version of the Liouville field:

$$
\varphi \equiv-\frac{1}{4} \log \left(\mathcal{I}_{11}^{2}-L^{2} \widehat{\mathcal{I}}_{22}\right)
$$

The operator $\widehat{\mathcal{I}}_{22}$ is a Weyl tensor of weight four, so the object $\varphi$ transforms as a scalar under worldsheet diffeomorphisms and as a Liouville field under Weyl transformations of the intrinsic metric:

$$
\varphi \rightarrow \varphi+\rho \quad \text { under } \quad g_{\bullet \bullet} \rightarrow \exp (2 \rho) g_{\bullet \bullet} .
$$

Therefore, the anomaly action evaluated on $\varphi$ has precisely the same anomaly-canceling property as the anomaly action evaluated with $L=0$, which leads to the PolchinskiStrominger anomaly term. In terms of $\varphi$, the regulated anomaly term can conveniently be expressed as [3]

$$
\mathcal{L}_{\text {anom }} \equiv \frac{\beta}{2 \pi}\left(-|\nabla \varphi|^{2}+\varphi R_{(2)}\right),
$$

where $R_{(2)}$ is the Ricci scalar curvature of the two-dimensional intrinsic metric.

After gauge-fixing $g_{a b} \rightarrow \eta_{a b}$, the anomaly term becomes

$$
\mathcal{L}_{\text {anom }}=\frac{2 \beta}{\pi} \partial_{+} \varphi \partial_{-} \varphi .
$$

The path integral with the addition of this term is fully gauge invariant and finite at the boundary.

We now turn to the evaluation of this term in the classical helical solution (A.1). For $p, q \leq 2$, the ground state profile of $\mathcal{I}_{p q}$ is as follows:

$$
\begin{aligned}
& \mathcal{I}_{11}=-2 \alpha^{\prime}\left(J_{1}+4 J_{2}+4 J_{2} \cos \left(2 \sigma_{1}\right)\right) \sin ^{2}\left(\sigma_{1}\right) \\
& \mathcal{I}_{12}=-\mathcal{I}_{21}=\alpha^{\prime}\left(J_{1}+8 J_{2} \cos \left(2 \sigma_{1}\right)\right) \sin \left(2 \sigma_{1}\right) \\
& \mathcal{I}_{22}=\alpha^{\prime}\left(J_{1} \cos \left(2 \sigma_{1}\right)+8 J_{2} \cos \left(4 \sigma_{1}\right)\right) .
\end{aligned}
$$


Taking these together, we recover an explicit expression for the Weyl-covariant version of $\mathcal{I}_{22}$ on the ground state solution

$$
\widehat{\mathcal{I}}_{22}=\alpha^{\prime}\left(J_{1} \cos \left(2 \sigma_{1}\right)+8 J_{2} \cos \left(4 \sigma_{1}\right)-2 \cos ^{2}\left(\sigma_{1}\right) \frac{\left(J_{1}+8 J_{2} \cos ^{2}\left(2 \sigma_{1}\right)\right)^{2}}{J_{1}+4 J_{2}+4 J_{2} \cos \left(2 \sigma_{1}\right)}\right) .
$$

Analysis of the PS anomaly contribution can thus be reduced to a straightforward contour integral evaluated by residues, with the removal of a UV divergence at the endpoints of the interval. Let us introduce the following change of variables:

$$
\sigma_{1}=\frac{1}{2 i} \log w, \quad d \sigma_{1}=\frac{1}{2 i} \frac{d w}{w} .
$$

We can infer the location of the poles of the PS integrand (as a function of $w$ ) by looking at the denominator of the PS anomaly term. To do this, let us further define

$$
a \equiv \frac{J_{2}}{J_{1}}, \quad b \equiv \frac{L}{J_{1}^{1 / 2}}
$$

and write the integrated Lagrangian as

$$
\int \mathcal{L}_{\mathrm{PS}} d \sigma_{1}=-i \frac{\beta}{4 \pi} \int \frac{(w+1)^{2}(w-1)^{6} F_{1}^{2}}{w\left[w+2 a(w+1)^{2}\right]^{2} F_{2}^{2}} d w
$$

The functions $F_{1}$ and $F_{2}$ are complicated polynomials in $w$, with coefficients depending on $a, b$ and $\alpha^{\prime}$. Organizing the polynomial coefficients according to

$$
\begin{aligned}
& F_{1}=\sum_{i=0}^{8} C_{1, i} w^{i}, \\
& F_{2}=\sum_{i=0}^{10} C_{2, i} w^{i},
\end{aligned}
$$

we have

$$
\begin{aligned}
& C_{1,0}=32 a^{4} \alpha^{\prime} \\
& C_{1,1}=8 a^{3}(7+24 a) \alpha^{\prime} \\
& C_{1,2}=4 a^{2}(a+4 a(15+32 a)) \alpha^{\prime} \\
& C_{1,3}=2 a\left[-2 a b^{2}+[5+4 a(12+a(57+104 a))] \alpha^{\prime}\right] \\
& C_{1,4}=-4 a(1+6 a) b^{2}+[1+4 a[3+2 a(15+4 a(17+30 a))]] \alpha^{\prime} \\
& C_{1,5}=2 a\left[-2 a b^{2}+[5+4 a(12+a(57+104 a))] \alpha^{\prime}\right] \\
& C_{1,6}=4 a^{2}(a+4 a(15+32 a)) \alpha^{\prime} \\
& C_{1,7}=8 a^{3}(7+24 a) \alpha^{\prime} \\
& C_{1,8}=32 a^{4} \alpha^{\prime}
\end{aligned}
$$


and

$$
\begin{aligned}
C_{2,0} & =8 a^{3} \alpha^{\prime} \\
C_{2,1} & =4 a^{2}(3+4 a) \alpha^{\prime} \\
C_{2,2} & =6 a\left(1-4 a^{2}\right) \alpha^{\prime} \\
C_{2,3} & =-4 a b^{2}-(-1+4 a(3+4 a(3+4 a))) \alpha^{\prime} \\
C_{2,4} & =8 a(3+8 a) b^{2}+2\left(-2-3 a+8 a^{3}\right) \alpha^{\prime} \\
C_{2,5} & =4\left(1+6 a+32 a^{2}\right) b^{2}+6(1+2 a)\left(1+2 a+8 a^{2}\right) \alpha^{\prime} \\
C_{2,6} & =8 a(3+8 a) b^{2}+2\left(-2-3 a+8 a^{3}\right) \alpha^{\prime} \\
C_{2,7} & =-4 a b^{2}-(-1+4 a(3+4 a(3+4 a))) \alpha^{\prime} \\
C_{2,8} & =6 a\left(1-4 a^{2}\right) \alpha^{\prime} \\
C_{2,9} & =4 a^{2}(3+4 a) \alpha^{\prime} \\
C_{2,10} & =8 a^{3} \alpha^{\prime} .
\end{aligned}
$$

The analysis of the contour integral can then be organized as follows. Poles (single or multiple) of the integrand can be sorted into those that give a nonzero contribution as $\sqrt{L} \rightarrow 0$, and those that give vanishing contributions as $\sqrt{L} \rightarrow 0$. In the second category, we find poles that lie outside the unit circle in the $w$ plane at sufficiently small $\sqrt{L}$, as well as poles that either disappear or exhibit vanishing residue as $\sqrt{L} \rightarrow 0$. In particular, any pole that approaches any point on the $w$ unit circle other than the point $w=1$ as $\sqrt{L} \rightarrow 0$ fall into the latter category; as $\sqrt{L} \rightarrow 0$ we must recover the original unregulated integrand, which is smooth everywhere on the unit circle except at the point $w=1$.

Among the poles that provide a nonzero contribution, we find a set of poles that approach points interior to the unit circle as $\sqrt{L} \rightarrow 0$, and a set that approaches the point $w=1$ in the same limit. Contributions from the former set can be computed by setting $\sqrt{L}$ to zero at the outset, identifying poles interior to the unit circle, and calculating the corresponding residues. Contributions from the second set can be determined by making the change of variables $w \rightarrow 1+i \sqrt{L} v$ and examining the limit $\sqrt{L} \rightarrow 0$ for fixed $v$. In this limit, the positions of the singularities approach fixed locations in the $v$ plane, and the residues scale ${ }^{12}$ as $L^{-1 / 2}$. That is, in the scaling limit $L \rightarrow 0$, the PS term in these variables takes the form

$$
\int \mathcal{L}_{\mathrm{PS}} d \sigma_{1}=L_{\mathrm{PS}}^{(\text {finite) }}+L_{\mathrm{PS}}^{(\text {divergent })}+O\left(L^{1 / 2}\right)
$$

where the divergent term comes entirely from the scaling limit of the integral near the cluster of poles near $w=1$ :

$$
L_{\mathrm{PS}}^{\text {(divergent) }} \equiv \int\left(-\frac{\beta \alpha^{\prime 2}}{\pi \sqrt{L}} \frac{q^{2} v^{6}}{\left(4+\alpha^{\prime} q v^{4}\right)^{2}}\right) d v
$$

\footnotetext{
${ }^{12}$ This scaling comes from a contribution of $\sqrt{L}$, strictly from the transformed measure, and a contribution of $L^{-1}$ from the leading-order scaling of the original integrand, modulo the measure.
} 
and $q$ is the combination

$$
q \equiv J_{1}+8 J_{2}
$$

The value of the divergent term is:

$$
L_{\mathrm{PS}}^{\text {(divergent) }}=-\frac{3 \beta\left(q \alpha^{\prime}\right)^{\frac{1}{4}}}{8 \sqrt{L}}
$$

Let us emphasize here that the $L^{-1 / 2}$ divergences are strictly proportional to the term $\mathcal{O}_{\text {quark }}=\mathcal{B}_{(22)}^{1 / 4}$. (Indeed, according to the dressing structure and, correspondingly, the allowed spectrum of boundary operators in the effective theory, this is the only possibility.) The particular combination $J_{1}+8 J_{2}$ occurring inside the fourth root agrees nontrivially with $\mathcal{B}_{(22)}$, which can be read, e.g., from the boundary value of $\mathcal{I}_{11}$ in (A.13). In the helical solution, this operator scales as $\left\langle\mathcal{O}_{\text {quark }}\right\rangle \propto\left(J_{1}+8 J_{2}\right)^{1 / 4}=q^{1 / 4}$, so the divergence of the PS integrand thus appears as $\left\langle\mathcal{O}_{\text {quark }}\right\rangle$ with a coefficient that diverges as $L^{-1 / 2}$. There are also terms in the integrand of order $\sqrt{L}^{0}$, but these turn out to be odd in $v$, and thus integrate to zero.

The finite terms come from poles interior to the unit circle in the $L \rightarrow 0$ limit, and the sum of their residues can be found by integrating along a circle enclosing all the poles away from $w \sim 1$, but excluding the poles near $w=1$. In the $L=0$ expression, the interior singularities comprise a single pole at the origin, and a double pole at

$$
w_{(*)}^{\text {int }} \equiv \frac{1}{4 J_{2}}\left(J_{1}^{1 / 2} \sqrt{J_{1}+8 J_{2}}-J_{1}-4 J_{2}\right),
$$

which is always real and lies between 0 and -1 .

Altogether, the integral decomposes into contributions that are manifestly regulatorindependent (i.e., those that approach interior points to the unit circle as $\sqrt{L} \rightarrow 0$ ), and contributions from a purely local UV divergence (i.e., the sum of contributions from poles that approach $w=1$ as $\sqrt{L} \rightarrow 0$ ). The contribution from the pole at the origin is

$$
\frac{2 \beta}{\pi} \oint_{w \rightarrow 0} \partial_{+} \varphi \partial_{-} \varphi d \sigma=2 \beta
$$

and the contribution from the double pole at $w_{(*)}^{\text {int }}$ is

$$
\frac{2 \beta}{\pi} \oint_{w \rightarrow w_{(*)}^{\text {int }}} \partial_{+} \varphi \partial_{-} \varphi d \sigma=-\frac{\beta}{2} \frac{3 J_{1}+4 J_{2}}{\sqrt{J_{1}\left(J_{1}+8 J_{2}\right)}} .
$$

Thus, the UV-finite part of the PS anomaly term (A.21) evaluates to

$$
L_{\mathrm{PS}}^{(\text {finite) }}=\frac{2 \beta}{\pi} \int \partial_{+} \varphi \partial_{-} \varphi d \sigma=\frac{\beta}{2}\left(4-\frac{3 J_{1}+4 J_{2}}{\sqrt{J_{1}\left(J_{1}+8 J_{2}\right)}}\right),
$$

while the divergent piece can be removed with a boundary counterterm proportional to the quark mass operator. As described in [4], the first-order shift in the energy of the lowest classical solution with fixed Noether charges is just the negative of the interaction 
Lagrangian for the unperturbed, zeroth-order helically symmetric solution. Replacing $\beta=$ $(26-D) / 12$ (see $[1,4]$ ), we recover the contribution to the open string mass-squared from the PS interaction:

$$
\Delta M_{\mathrm{open}}^{2}=\frac{D-26}{24 \alpha^{\prime}}\left(4-\frac{3 J_{1}+4 J_{2}}{\sqrt{J_{1}\left(J_{1}+8 J_{2}\right)}}\right)
$$

\section{B Properties of geodesics near the boundary}

In the displaced-boundary regulator analysis of section 4, we introduced a near-boundary cutoff scheme by defining a strip to be excised from the worldsheet along a set of points on the $\sigma_{1}=\tilde{\sigma}$ locus separated from the boundary by a fixed geodesic distance $\epsilon$. Here we demonstrate in detail the gauge-invariant characterization of this distance function. In particular, we identify the longest ${ }^{13}$ spacelike geodesic in the near-boundary region, extending from the boundary to the interior point $\tilde{\sigma}$. We can show that such a global maximum must always exist by first anchoring a point in the bulk. Given a point on the boundary, there is always a geodesic of some kind from the anchor point to that boundary point. For boundary points sufficiently far in the past or the future, the geodesic will be future-oriented timelike or past-oriented timelike. Between, it must necessarily be spacelike. The geodesic length varies continuously across this region of the boundary, so it must assume a global maximum. (On the endpoints of the spacelike-separated region, the proper length goes to zero, so the maximum is never assumed at the endpoints.) For the static geometry induced by the helical solution, this geodesic is just the horizontal trajectory in the obvious flat coordinates.

Let us now make this argument more concrete. With the expansion of $\mathcal{I}_{11}$ in the near-boundary region (3.11), we can characterize the form of the metric in this region as

$$
\widehat{d s^{2}} \equiv \frac{d s^{2}}{\mathcal{B}_{(22)}}=\sigma_{1}^{2}\left(-d \sigma_{0}^{2}+d \sigma_{1}^{2}\right)
$$

It is convenient to make the following change of variables:

$$
a \equiv \frac{\sigma_{1}^{2}}{2} .
$$

We can always parameterize sufficiently short geodesics as functions of $\sigma_{1}$, so, in turn, we define

$$
b \equiv \sqrt{2} \sigma^{0} \equiv h(a)
$$

Working up to an overall scaling of the metric, we have

$$
\widehat{d s^{2}}=d a^{2}-a d b^{2}=d a^{2}-a\left(h^{\prime}\right)^{2} d a^{2}=\left(1-a\left(h^{\prime}\right)^{2}\right) d a^{2} .
$$

The arc length is then

$$
\hat{\ell}=\int \sqrt{1-a\left(h^{\prime}\right)^{2}} d a
$$

\footnotetext{
${ }^{13}$ In Lorentzian signature.
} 
such that the geodesic equation on this space is just

$$
0=\partial_{a}\left(\frac{a h^{\prime}}{\sqrt{1-a\left(h^{\prime}\right)^{2}}}\right) \rightarrow \frac{a h^{\prime}}{\sqrt{1-a\left(h^{\prime}\right)^{2}}}=K,
$$

where $K$ is a constant. Solving for $\left(h^{\prime}\right)^{2}$,

$$
\left(h^{\prime}\right)^{2}=\frac{K^{2}}{a\left(a+K^{2}\right)},
$$

the geodesic equation admits solutions of the form

$$
h(a)=\text { const. } \pm 2 K \log \left(\sqrt{a}+\sqrt{a+K^{2}}\right) .
$$

When $K$ is nonzero, at small $\sigma_{1}$ (correspondingly, at small $a$ ), the solutions are of the form

$$
\left.h(a)\right|_{a \text { small }} \approx \text { const. }+\left(\text { linear in } \sigma_{1}\right)+\cdots
$$

As we approach the boundary, solutions with vanishing $K$ are asymptotically purely spacelike and normal to the boundary.

One concern might have been that the singularity at the boundary might spoil this analysis, though it does not. The integrated geodesic length remains finite, for instance, as a function of the natural conformal coordinate $\sigma_{1}$.

More directly, we can compute the arc length of these geodesics from the boundary to an anchor point $a_{0}$ in the interior. We obtain

$$
\begin{aligned}
\hat{\ell} & =\int_{0}^{a_{0}} \sqrt{1-\frac{K^{2}}{a+K^{2}}} d a \\
& =\sqrt{a_{0}\left(a_{0}+K^{2}\right)}+K^{2} \log \left(\frac{K}{\sqrt{a_{0}}+\sqrt{a_{0}+K^{2}}}\right) .
\end{aligned}
$$

It can be shown that the $K=0$ class of solutions globally maximizes the length function in the asymptotic near-boundary region. In detail, the first derivative of $l$ with respect to $K$ is

$$
\begin{aligned}
\frac{\partial \hat{\ell}}{\partial K} & =2 K\left(\sqrt{\frac{a_{0}}{a_{0}+K^{2}}}+\log \left(\frac{K}{\sqrt{a_{0}}+\sqrt{a_{0}+K^{2}}}\right)\right) \\
& \approx\left(2-\log \left(4 a_{0}\right)+2 \log (K)\right) K-\frac{3 K^{3}}{2 a_{0}}+O\left(K^{5}\right),
\end{aligned}
$$

which vanishes in the limit $K \rightarrow 0$. The second derivative

$$
\begin{aligned}
\frac{\partial^{2} \hat{\ell}}{\partial K^{2}} & =2\left(\frac{\sqrt{a_{0}}\left(2 a_{0}+K^{2}\right)}{\left(a_{0}+K^{2}\right)^{3 / 2}}+\log K-\log \left(\sqrt{a_{0}}+\sqrt{a_{0}+K^{2}}\right)\right) \\
& \approx 4-\log \left(4 a_{0}\right)+2 \log K-\frac{9 K^{2}}{2 a_{0}}+O\left(K^{4}\right)
\end{aligned}
$$

is negative as $K \rightarrow 0$. 


\section{Acknowledgments}

The authors are deeply grateful to J. Sonnenschein and O. Aharony for discussions that were responsible for refining the ideas presented herein and motivating the derivation of the dressing rule in section 3. The work of SH is supported by the World Premier International Research Center Initiative (WPI Initiative), MEXT, Japan; by the JSPS Program for Advancing Strategic International Networks to Accelerate the Circulation of Talented Researchers; and also supported in part by JSPS KAKENHI Grant Numbers JP22740153, JP26400242. SH is also grateful to the CCPP and New York University and the Walter Burke Institute for Theoretical Physics at Caltech for generous hospitality while this work was in progress.

Open Access. This article is distributed under the terms of the Creative Commons Attribution License (CC-BY 4.0), which permits any use, distribution and reproduction in any medium, provided the original author(s) and source are credited.

\section{References}

[1] J. Polchinski and A. Strominger, Effective string theory, Phys. Rev. Lett. 67 (1991) 1681 [INSPIRE].

[2] N.D. Hari Dass and P. Matlock, Covariant Calculus for Effective String Theories, Indian J. Phys. 88 (2014) 965 [arXiv:0709.1765] [InSPIRE].

[3] S. Hellerman, S. Maeda, J. Maltz and I. Swanson, Effective String Theory Simplified, JHEP 09 (2014) 183 [arXiv: 1405.6197] [inSPIRE].

[4] S. Hellerman and I. Swanson, String Theory of the Regge Intercept, Phys. Rev. Lett. 114 (2015) 111601 [arXiv: 1312.0999] [INSPIRE].

[5] O. Aharony and M. Field, On the effective theory of long open strings, JHEP 01 (2011) 065 [arXiv: 1008.2636] [INSPIRE].

[6] O. Aharony and Z. Komargodski, The Effective Theory of Long Strings, JHEP 05 (2013) 118 [arXiv: 1302.6257] [INSPIRE].

[7] O. Aharony, The effective action on the confining string, presented at The Strings 2009 Conference, June 2009, http://strings2009.roma2.infn.it/talks/Aharony_Strings09.ppt.

[8] B.B. Brandt and P. Majumdar, Spectrum of the QCD flux tube in 3d $\mathrm{SU}(2)$ lattice gauge theory, Phys. Lett. B 682 (2009) 253 [arXiv:0905.4195] [INSPIRE].

[9] B.B. Brandt, Probing boundary-corrections to Nambu-Goto open string energy levels in $3 d$ SU(2) gauge theory, JHEP 02 (2011) 040 [arXiv: 1010.3625] [INSPIRE].

[10] B.B. Brandt, Spectrum of the open QCD flux tube in $d=2+1$ and its effective string description, PoS (EPS-HEP 2013) 540 [arXiv: 1308.4993] [INSPIRE].

[11] B.B. Brandt and M. Meineri, Effective string description of confining flux tubes, Int. J. Mod. Phys. A 31 (2016) 1643001 [arXiv:1603.06969] [InSPIRE].

[12] M. Baker and R. Steinke, Semiclassical quantization of effective string theory and Regge trajectories, Phys. Rev. D 65 (2002) 094042 [hep-th/0201169] [INSPIRE].

[13] F. Wilczek, Diquarks as inspiration and as objects, hep-ph/0409168 [INSPIRE]. 
[14] J. Sonnenschein and D. Weissman, Rotating strings confronting PDG mesons, JHEP 08 (2014) 013 [arXiv: 1402.5603] [INSPIRE].

[15] O. Ganor, J. Sonnenschein and S. Yankielowicz, Folds in 2-D string theories, Nucl. Phys. B 427 (1994) 203 [hep-th/9404149] [INSPIRE].

[16] S. Caron-Huot, Z. Komargodski, A. Sever and A. Zhiboedov, Strings from Massive Higher Spins: The Asymptotic Uniqueness of the Veneziano Amplitude, arXiv:1607.04253 [INSPIRE].

[17] J. Sonnenschein and D. Weissman, Glueballs as rotating folded closed strings, JHEP 12 (2015) 011 [arXiv: 1507.01604] [INSPIRE].

[18] G. German and Y. Jiang, On quark mass correction to the string potential, Eur. Phys. J. C 8 (1999) 689 [hep-th/9707037] [INSPIRE]. 